\title{
GENOMIC TABLEAUX
}

\author{
OLIVER PECHENIK AND ALEXANDER YONG
}

\begin{abstract}
We explain how genomic tableaux [Pechenik-Yong '15] are a semistandard complement to increasing tableaux [Thomas-Yong '09]. From this perspective, one inherits genomic versions of jeu de taquin, Knuth equivalence, infusion and Bender-Knuth involutions, as well as Schur functions from (shifted) semistandard Young tableaux theory. These are applied to obtain new Littlewood-Richardson rules for $K$-theory Schubert calculus of Grassmannians (after [Buch '02]) and maximal orthogonal Grassmannians (after [CliffordThomas-Yong '14], [Buch-Ravikumar '12]). For the unsolved case of Lagrangian Grassmannians, sharp upper and lower bounds using genomic tableaux are conjectured.
\end{abstract}

\section{CONTENTS}

1. Introduction 2

1.1. History and overview

1.2. Genomic tableau results 3

1.3. Genomic rules in Schubert calculus 4

2. K-(semi)standardization maps 5

3. Genomic words and Knuth equivalence 7

4. Genomic jeu de taquin 8

5. Three proofs of the Genomic Littlewood-Richardson rule (Theorem 1.4) 10

5.1. Proof 1: Bijection with increasing tableaux 10

5.2. Proof 2: Bijection with set-valued tableaux 11

5.3. Proof 3: Bijection with puzzles 13

6. Infusion, Bender-Knuth involutions and the genomic Schur function 14

7. Shifted genomic tableaux 15

8. Maximal orthogonal and Lagrangian Grassmannians 18

9. Proof of OG Genomic Littlewood-Richardson rule (Theorem 8.1) 21

9.1. Shifted $K$-(semi)standardization maps 21

9.2. Genomic $P$-Knuth equivalence 23

9.3. Shifted jeu de taquin and the conclusion of the proof 27

Acknowledgments $\quad 28$

\begin{tabular}{ll} 
References & 28 \\
\hline
\end{tabular}

Date: March 28, 2016. 


\section{INTRODUCTION}

1.1. History and overview. Let Sym be the ring of symmetric functions. Textbook theory of Sym concerns the basis of Schur functions and the package of Young tableau algorithms for which the Littlewood-Richardson rule is a centerpiece. Interpreting these polynomials in the Schubert calculus of Grassmannians one is led, via a $K$-theoretic generalization, to symmetric Grothendieck functions $\left\{G_{\lambda}\right\}$, a deformation of the Schur basis $\left\{s_{\lambda}\right\}$. This line of inquiry started with [LaSc82]. The first combinatorial rule for $G_{\lambda}$ was given by [FoKi94] whereas the first tableau formula was found by [Bu02].

There is interest in finding $K$-analogues of elements of the classical Young tableau theory; see, e.g., [Le00, Bu02, BKSTY08, ThYo09b, BuSa13, GMPPRST16, PaPy14, HKPWZZ15, LiMoSh16]. Although the Grothendieck functions were originally studied for geometric reasons, the combinatorics has been part of a broader conversation in algebraic and enumerative combinatorics, e.g., Hopf algebras [LaPy07, PaPy16, Pa15], cyclic sieving [Pe14, Rh15, PrStVi14], Demazure characters [Mo16+], homomesy [BlPeSa16], longest increasing subsequences of random words [ThYo11], poset edge densities [ReTeYo16], and plane partitions [DiPeSt15, HPPW16].

In [ThYo09b], a jeu de taquin theory for increasing tableaux was introduced. These tableaux are fillings of Young diagrams $\nu / \lambda$ with $[\ell]:=1,2, \ldots, \ell$ where $\ell \leq|\nu / \lambda|$ and the entries increase in rows and columns (labels may be repeated). If $\ell=|\nu / \lambda|$, these are standard Young tableaux and increasing tableau results closely parallel those for standard Young tableaux. An outcome was a new Littlewood-Richardson rule for $\left\{G_{\lambda}\right\}$ (after [Bu02]) and its minuscule extension (see [ThYo09a, BuRa12, ClThYo14, BuSa13]).

In [ThYo13], a jeu de taquin-based Littlewood-Richardson rule for torus-equivariant $K$ theory of Grassmannians was conjectured. In [PeYo15b], we proved this conjecture by defining genomic tableaux as a semistandard analogue of increasing tableaux.

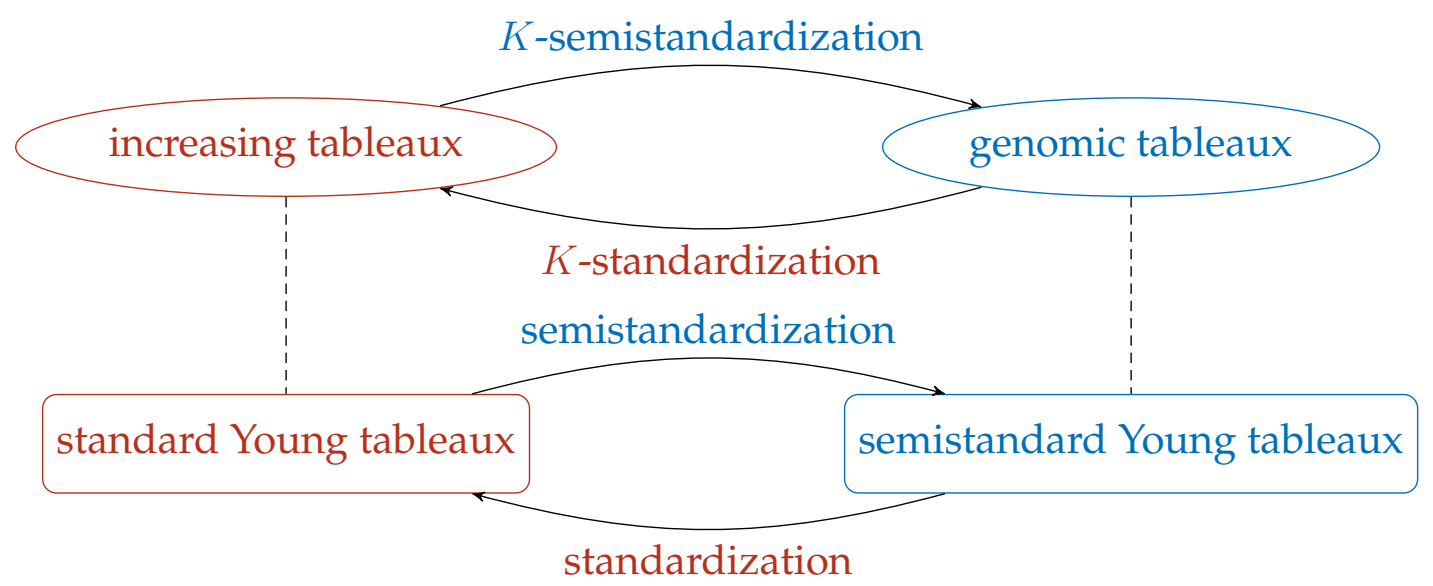

Our goal is a theory of genomic tableaux parallel to that of [ThYo09] for increasing tableaux. The Schubert calculus application in [PeYo15b, PeYo15c] used edge-labeled genomic tableaux. However, in anticipation of other applications, we give a logically independent development of genomic tableau combinatorics in the basic (i.e., non-edge 
labeled) case and in the shifted setting. The first applications are to give new LittlewoodRichardson-type rules for (ordinary) $K$-theory of Grassmannians and maximal orthogonal Grassmannians. Furthermore, modifications of these rules give conjectural upper and lower bounds for the $K$-theory structure constants of Lagrangian Grassmannians.

1.2. Genomic tableau results. Let $S$ be a semistandard Young tableau of a shape $\nu / \lambda$. Place a total order on those boxes with entry $i$ using left to right order. A gene $\mathcal{G}$ (of family $i$ ) is a collection of consecutive boxes in this order, where no two lie in the same row; we write $\operatorname{family}(\mathcal{G})=i$. A genomic tableau $T$ is a semistandard tableau together with a partition of its boxes into genes. We indicate the partition by color-coding the boxes. The content of $T$ is the number of genes of each family. Note, a semistandard tableau $T$ is a genomic tableau where each gene is a single box. Moreover, the content of $T$ agrees with the usual notion for semistandard tableaux.

Example 1.1. $T=$\begin{tabular}{|l|l|l|l|}
\hline & & 1 & 2 \\
\hline 1 & 1 & 2
\end{tabular}$\quad$ has content $(2,1)$ since there are two genes of family 1 and one of family 2 .

A genotype $G$ of a genomic tableau $T$ is a choice of a single box from each gene 1 We depict $G$ by erasing the entries in all unchosen boxes of $T$.

Example 1.2. Continuing Example 1.1,
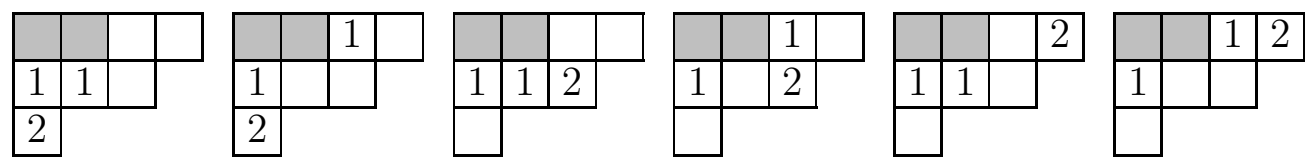

are the six genotypes of $T$.

Suppose $U$ is any filling of a subset of boxes of a shape. The sequence $\operatorname{seq}(U)$ of $U$ is the reading word obtained by reading its entries along rows from right to left and from top to bottom (ignoring empty boxes). Now, $\mathrm{seq}(U)$ is a ballot sequence if the number of $i$ 's that appear is always weakly greater than the number of $(i+1)$ 's that appear, at any point in the sequence. A genomic tableau $T$ is ballot if $\operatorname{seq}(G)$ is a ballot sequence for every genotype $G$ of $T$. Notice if each gene of $T$ is a single box, there is a unique genotype (namely, the underlying semistandard tableau of $T$ ) and the concept of a ballot tableau coincides with the same notion for semistandard tableaux.

Example 1.3. The genotypes of Example 1.2 respectively have sequences: 112, 112, 211, 121,211 , and 211. Since 211 is not a ballot sequence, $T$ is not ballot.

Our results are:

(1) A $K$-analogue of the (semi)standardization maps between standard and semistandard tableaux. This relates genomic tableaux to increasing tableaux.

(2) Using (1), we acquire genomic analogues of Knuth equivalence, jeu de taquin, infusion and Bender-Knuth involutions.

(3) Using (2), we describe a new basis $\left\{U_{\lambda}\right\}$ of Sym where each $U_{\lambda}$ is a generating series over genomic tableaux of shape $\lambda$. This is a deformation of the Schur basis.

(4) We give shifted analogues of (1)-(3).

${ }^{1}$ The genomic analogy is that boxes of a gene are alleles and the other genes of the same family are paralogs. 


\subsection{Genomic rules in Schubert calculus. Let}

$$
X=\operatorname{Gr}_{k}\left(\mathbb{C}^{n}\right)
$$

denote the Grassmannian of $k$-dimensional linear subspaces of $\mathbb{C}^{n}$. The general linear group $\mathrm{GL}_{n}$, and its Borel subgroup $\mathrm{B}_{-}$of lower triangular invertible matrices, act on $X$ by change of basis. This action decomposes $X$ into $\mathrm{B}_{-}$-orbits

$$
X_{\lambda}^{\circ} \cong \mathbb{C}^{k(n-k)-|\lambda|}
$$

(the Schubert cells); here $\lambda$ is a Young diagram contained in the rectangle $k \times(n-k)$. The Zariski closure of $X_{\lambda}^{\circ}$ is the Schubert variety

$$
X_{\lambda}=\bigsqcup_{\mu \supseteq \lambda} X_{\mu}^{\circ}
$$

Textbook discussion of Schubert calculus revolves around classes of $X_{\lambda}$ in the cohomology ring $H^{\star}(X, \mathbb{Z})$; see, e.g., [Fu97]. These classes form a $\mathbb{Z}$-linear basis of $H^{\star}(X, \mathbb{Z})$. Their structure constants

$$
\left[X_{\lambda}\right] \cup\left[X_{\mu}\right]=\sum_{\nu} C_{\lambda, \mu}^{\nu}\left[X_{\nu}\right]
$$

with respect to the cup product are given by the classical Littlewood-Richardson rule that governs the multiplication of Schur functions. Geometrically,

$$
C_{\lambda, \mu}^{\nu}=\text { \#points in } g_{1} \cdot X_{\lambda} \cap g_{2} \cdot X_{\mu} \cap g_{3} \cdot X_{\nu^{\vee}}
$$

for generic $g_{1}, g_{2}, g_{3} \in \mathrm{GL}_{n}$ when this number is finite; it is zero otherwise. Here,

$$
\nu^{\vee}=\left(n-k-\nu_{k}, n-k-\nu_{k-1}, \ldots, n-k-\nu_{1}\right)
$$

is the rotation of the complement of $\nu$ in $k \times(n-k)$.

There has been significant attention on $K$-theoretic Schubert calculus, which provides a richer setting for study; see, e.g., [Br05, Bu05, Va06, Kn14] and the references therein. Recall that the Grothendieck ring $K^{0}(X)$ is the free abelian group generated by isomorphism classes $[V]$ of algebraic vector bundles over $X$ under the relation

$$
[V]=[U]+[W]
$$

whenever there is a short exact sequence

$$
0 \rightarrow U \rightarrow V \rightarrow W \rightarrow 0
$$

The product structure on $K^{0}(X)$ is given by the tensor product of vector bundles, i.e.,

$$
[U] \cdot[V]=[U \otimes V] .
$$

Since $X$ is a smooth projective variety, the structure sheaf $\mathcal{O}_{X_{\lambda}}$ has a resolution

$$
0 \rightarrow V_{N} \rightarrow V_{N-1} \rightarrow \cdots \rightarrow V_{1} \rightarrow V_{0} \rightarrow \mathcal{O}_{X_{\lambda}} \rightarrow 0
$$

by locally free sheaves. Therefore it makes sense to define the class $\left[\mathcal{O}_{X_{\lambda}}\right]$ by

$$
\left[\mathcal{O}_{X_{\lambda}}\right]:=\sum_{j=0}^{N}(-1)^{j}\left[V_{j}\right] \in K^{0}(X) .
$$

Now, $\left\{\left[\mathcal{O}_{X_{\lambda}}\right]\right\}$ forms a $\mathbb{Z}$-linear basis of $K^{0}(X)$. Thus, define structure constants by

$$
\left[\mathcal{O}_{X_{\lambda}}\right] \cdot\left[\mathcal{O}_{X_{\mu}}\right]=\sum_{\nu} a_{\lambda, \mu}^{\nu}\left[\mathcal{O}_{X_{\nu}}\right]
$$


A. Buch [Bu02] gave a combinatorial rule for $a_{\lambda, \mu}^{\nu}$, thereby establishing

$$
(-1)^{|\nu|-|\lambda|-|\mu|} a_{\lambda, \mu}^{\nu} \geq 0 \text {. }
$$

A number of other rules have been discovered since, see, e.g., [Va06, BKSTY08, ThYo09b] and the references therein, as well as the references above.

Theorem 1.4 (Genomic Littlewood-Richardson rule). $a_{\lambda, \mu}^{\nu}=(-1)^{|\nu|-|\lambda|-|\mu|}$ times the number of ballot genomic tableaux of shape $\nu / \lambda$ and content $\mu$.

Actually, in the case $|\nu|=|\lambda|+|\mu|, a_{\lambda, \mu}^{\nu}=C_{\lambda, \mu}^{\nu}$ and Theorem 1.4 recovers the original rule of D.E. Littlewood-A.R. Richardson for multiplication of Schur functions [LiRi34].

Example 1.5. The tableau

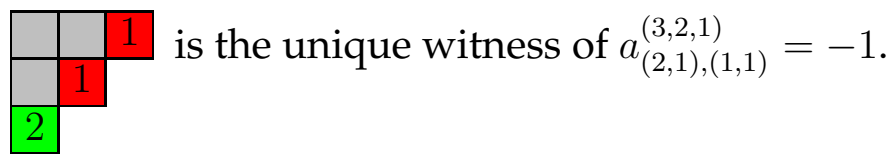

Using the tableau results outlined in Section 1.2, the proof of Theorem 1.4 is derived from the corresponding Littlewood-Richardson rule in [ThYo09b].

Similarly, using our theorems on shifted genomic tableaux, we obtain a combinatorial rule for the $K$-theoretic structure constants for maximal orthogonal Grassmannians from the corresponding theorem in [ClThYo14] (originally conjectured in [ThYo09b]).

In cohomology, there is a simple relation between the structure constants for maximal orthogonal and Lagrangian Grassmannians. Hence, given the aforementioned results, it may come as a bit of surprise that there is no known combinatorial rule for the $K$ theory structure constants of Lagrangian Grassmannians. We use genomic tableaux to contribute related conjectural upper and lower bounds for these numbers. We further conjecture an inequality between the structure constants of Lagrangian Grassmannians and the maximal orthogonal Grassmannians.

\section{K-(SEMI)STANDARDIZATION MAPS}

Let

$$
\operatorname{Gen}_{\mu}(\nu / \lambda)=\left\{\text { genomic tableaux of shape } \nu / \lambda \text { with content } \mu=\left(\mu_{1}, \mu_{2} \ldots, \mu_{\ell(\mu)}\right)\right\},
$$

and

$$
\operatorname{Inc}(\nu / \lambda)=\{\text { increasing tableaux of shape } \nu / \lambda\} \text {. }
$$

Define an order on the genes of $T \in \operatorname{Gen}_{\mu}(\nu / \lambda)$ by $\mathcal{G}_{1}<\mathcal{G}_{2}$ if $\operatorname{family}\left(\mathcal{G}_{1}\right)<\operatorname{family}\left(\mathcal{G}_{2}\right)$ or if $\operatorname{family}\left(\mathcal{G}_{1}\right)=\operatorname{family}\left(\mathcal{G}_{2}\right)$ with all boxes of $\mathcal{G}_{1}$ west of all boxes of $\mathcal{G}_{2}$.

Lemma 2.1. The order $<$ on genes of $T$ is a total order.

Proof. When showing two genes $\mathcal{G}_{1}$ and $\mathcal{G}_{2}$ are comparable in the order $<$, the only concern is if $\operatorname{family}\left(\mathcal{G}_{1}\right)=\operatorname{family}\left(\mathcal{G}_{2}\right)=k$. By definition, a gene of family $k$ consists of boxes of entry $k$ that are consecutive in the left to right order on such boxes. Hence either all boxes of $\mathcal{G}_{1}$ are west of the boxes of $\mathcal{G}_{2}$ or vice versa.

The $K$-standardization map,

$$
\Phi: \operatorname{Gen}_{\mu}(\nu / \lambda) \rightarrow \operatorname{Inc}(\nu / \lambda)
$$


is defined by filling the $k$ th gene in the $<$-order with the entry $k$. Since any $T \in \operatorname{Gen}_{\mu}(\nu / \lambda)$ is also a semistandard tableau (by forgetting the gene structure) and since no two boxes of the same gene can be in the same row, it follows that $\Phi(T) \in \operatorname{Inc}(\nu / \lambda)$. Note that when each gene is a single box, $\Phi$ is the usual standardization map.

Example 2.2. If $T$ is the genomic tableau

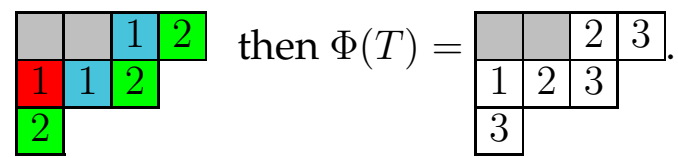

A horizontal strip is a skew shape with no two boxes in the same column. Following [ThYo09b], a Pieri filling is an increasing tableau of horizontal strip shape where, in addition, labels weakly increase from southwest to northeast.

Let

$$
\mathcal{P}_{k}(\mu):=\left\{1+\sum_{i<k} \mu_{i}, 2+\sum_{i<k} \mu_{i}, \ldots, \sum_{j \leq k} \mu_{j}\right\} .
$$

That is,

$$
\mathcal{P}_{1}(\mu)=\left\{1,2, \ldots, \mu_{1}\right\}, \mathcal{P}_{2}(\mu)=\left\{\mu_{1}+1, \ldots, \mu_{1}+\mu_{2}\right\}, \text { etc. }
$$

We say $S \in \operatorname{Inc}(\nu / \lambda)$ is $\mu$-Pieri-filled if for each $k \leq \ell(\mu)$, the entries of $S$ in $\mathcal{P}_{k}(\mu)$ form a Pieri filling of a horizontal strip.

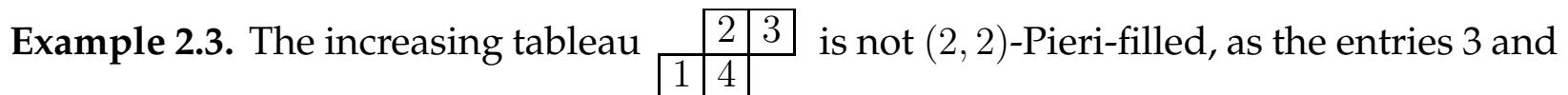
\begin{tabular}{|l|l|l|l|l}
\hline 1 & 3 & \\
\hline
\end{tabular}

4 do not form a Pieri filling. However, it is $(2,1,1)$-Pieri-filled.

Let

$$
\operatorname{PF}_{\mu}(\nu / \lambda)=\{S \in \operatorname{Inc}(\nu / \lambda) \text { that are } \mu \text {-Pieri-filled }\} .
$$

Theorem 2.4. $\Phi: \operatorname{Gen}_{\mu}(\nu / \lambda) \rightarrow \mathrm{PF}_{\mu}(\nu / \lambda)$ is a bijection.

Proof. We begin by defining the $K$-semistandardization map

$$
\Psi: \mathrm{PF}_{\mu}(\nu / \lambda) \rightarrow \operatorname{Gen}_{\mu}(\nu / \lambda) .
$$

This extends the classical semistandardization map from standard Young tableaux to semistandard Young tableaux. Suppose $S \in \mathrm{PF}_{\mu}(\nu / \lambda)$. Construct a filling $T$ of $\nu / \lambda$ by placing into each box the unique positive integer $k$ such that $i \in \mathcal{P}_{k}(\mu)$, where $i$ is the entry of the corresponding box of $S$. Clearly, $T$ is a semistandard tableau.

Declare boxes of $T$ to be in the same gene if and only if the corresponding boxes of $S$ contain the same value. Since $S$ is an increasing tableau, each gene of $T$ has at most one box in any row. Since the entries of $S$ in $\mathcal{P}_{k}(\mu)$ form a Pieri filling, given any two genes $\mathcal{G}_{1}, \mathcal{G}_{2}$ of family $k$ in $T$, every box $\mathcal{G}_{1}$ appears west of every box of $\mathcal{G}_{2}$ (or vice versa). Hence $T \in \operatorname{Gen}_{\mu}(\nu / \lambda)$.

We now show that $\Phi$ is well-defined, i.e.,

$$
\operatorname{im} \Phi \subseteq \mathrm{PF}_{\mu}(\nu / \lambda) .
$$

Fix

and set

$$
T \in \operatorname{Gen}_{\mu}(\nu / \lambda)
$$

$$
S:=\Phi(T) \in \operatorname{Inc}(\nu / \lambda) .
$$


Let $\gamma \subseteq \nu / \lambda$ be the set of boxes that contain $k$ in $T$. By (column) semistandardness of $T, \gamma$ is a horizontal strip. Since $\Phi$ puts the labels of $\mathcal{P}_{k}(\mu)$ into $\gamma$ (in $S$ ) so as to increase southwest to northeast, the resulting filling is $\mu$-Pieri-filled.

By construction we have that

$$
\Phi \circ \Psi=\operatorname{id}_{\mathrm{PF}_{\mu}(\nu / \lambda)} \text { and } \Psi \circ \Phi=\operatorname{id}_{\operatorname{Gen}_{\mu}(\nu / \lambda)} .
$$

It is straightforward from the definitions that $\Psi$ are $\Phi$ are injective maps. Therefore, we conclude that $\Phi$ and $\Psi$ are mutually inverse bijections.

\section{GENOMIC WORDS AND KNUTH EQUIVALENCE}

A genomic word is a word $s$ of colored positive integers such that all $i$ 's of a fixed color are consecutive among the set of all $i$ 's. A genotype of $s$ is a subword that selects one letter of each color. Say $s$ is ballot if every genotype of $s$ is ballot.

Example 3.1. 212112 is a genomic word, whereas 212112 is not because the subword of 1's is 111 and the 1's are not consecutive.

Let genomicseq $(T)$ be the colored row reading word (taken in right to left and top to bottom order) of a genomic tableau $T$.

Lemma 3.2. For a genomic tableau $T$, genomicseq $(T)$ is a genomic word.

Proof. The follows from the semistandardness of $T$ together with the condition that the boxes of each gene of family $i$ are consecutive in the left-to-right order on $i^{\prime}$ s in $T$.

We extend the $K$-standardization map $\Phi$ to genomic words by $\Phi(s):=\operatorname{seq}(\Phi(\widehat{T}(s)))$ where $\widehat{T}(s)$ is the antidiagonal of disconnected boxes filled from northeast to southwest by the given genomic word.

\section{Lemma 3.3.}

(I) Every genomic word $s$ is genomicseq $(T)$ for some genomic tableau $T$.

(II) $T$ is ballot if and only if genomicseq $(T)$ is ballot.

(III) If genomicseq $(T)=s$, then $\Phi(s)=\operatorname{seq}(\Phi(T))$.

Proof. For (I), in particular, one can take $T=\widehat{T}(s)$. (II) is clear. (III) is straightforward.

Example 3.4. If $T$ is the genomic tableau \begin{tabular}{|l|l|l|l|}
\cline { 2 - 4 } & & 1 & 2 \\
\cline { 2 - 3 } & 1 & 1 & 2 \\
\hline 2
\end{tabular}

selecting one green letter, one red letter, and one blue letter from 212112 we arrive at three possible genotypes of genomicseq $(T): 211,121$ and 112 . Thus genomicseq $(T)$ is not ballot.

Genomic Knuth equivalence is the equivalence relation $\equiv_{G}$ on genomic words obtained as the transitive closure of

$$
\begin{aligned}
\mathbf{u} i i \mathbf{v} & \equiv_{G} \mathbf{u} i \mathbf{v}, \\
\mathbf{u} i j i \mathbf{v} & \equiv_{G} \mathbf{u} j i j \mathbf{v}, \\
\mathbf{u} j i k \mathbf{v} & \equiv_{G} \mathbf{u} j k i \mathbf{v}, \\
\mathbf{u} p q j \mathbf{v} & \equiv_{G} \mathbf{u} q p j \mathbf{v},
\end{aligned}
$$


where $i \leq j<k, p<j \leq q$, and red, blue, green are distinct colors. This equivalence relation is a genomic version of the $K$-Knuth equivalence introduced by A. Buch-M. Samuel [BuSa13, §5]. It furthermore generalizes Knuth equivalence [Kn70] in the sense that it agrees with this older notion on words where each letter is of a distinct color, obviating (G.1) and (G.2).

Theorem 3.5. If $\mathbf{x} \equiv_{G} \mathbf{y}$, then $\mathbf{x}$ is ballot if and only if $\mathbf{y}$ is ballot.

Proof. Let $\mathrm{x}$ be a genomic word. It suffices to show that (G.1)-(G.4) do not change the ballotness of $\mathrm{x}$.

(G.1) and (G.2) preserve the set of genotypes and therefore ballotness.

(G.3) clearly preserves ballotness unless $k=i+1$. In this case, since $i \leq j<k$, this means $i=j$. Suppose therefore

$$
\mathbf{x}=\mathbf{u} j j k \mathbf{v}, \text { and that } \mathbf{y}=\mathbf{u} j k j \mathbf{v} .
$$

Clearly if $\mathrm{x}$ is not ballot, then $\mathrm{y}$ is not ballot. Conversely, assume $\mathrm{x}$ is ballot. It is enough to show that $\mathbf{u} j k$ is ballot. Since $\mathbf{x}$ is ballot, the initial segment $\mathbf{u} j$ is ballot. Now, deleting the last letter of a ballot word leaves a ballot word. Since the last letter in the case at hand is $j$ it follows that the subsequence of $\mathbf{u} j$ formed by deleting every $j$ is ballot. In particular, every genotype of $\mathbf{u} j$ has strictly more $j^{\prime}$ s than $k^{\prime}$ s. Thus $\mathbf{u} j k$ (and hence $\mathbf{y}$ ) is ballot.

(G.4) is only a concern if $q=p+1$. In this case, since $p<j \leq q$, we must also have $j=q$. Thus,

$$
\mathbf{x}=\mathbf{u} p q q \mathbf{v} \text { and } \mathbf{y}=\mathbf{u} q p q \mathbf{v} .
$$

If $\mathbf{y}$ is ballot, then $\mathbf{x}$ is ballot. Conversely, assume $\mathbf{x}$ is ballot. It suffices to show that $\mathbf{u} q p$ is ballot. Since it is an initial segment of $\mathbf{x}, \mathbf{u} p q q$ is ballot. Given any two genes of family $q$ in any genomic word, one appears entirely right of the other. Thus $q$ does not appear in $\mathbf{u}$, and hence every genotype of $\mathbf{u} p q$ has strictly more $p^{\prime}$ s than $q^{\prime} \mathbf{s}$. Thus $\mathbf{u} q p$ is ballot.

\section{GENOMIC JEU DE TAQUIN}

If $T \in \operatorname{Gen}_{\mu}(\nu / \lambda)$, an inner corner of $T$ is a maximally southeast box of $\lambda$. Let $I$ be any set of inner corners of $T$. We obtain a genomic tableau $j \mathrm{dt}_{I}(T)$ as follows: Place a $\bullet$ in each box of $I$; let $T^{\bullet}$ denote the result. Two boxes of a tableau are neighbors if they share a horizontal or vertical edge. For each gene $\mathcal{G}$, define the operator switch $_{\mathcal{G}}^{\bullet}$ as follows. Every box of $\mathcal{G}$ with a neighbor containing a $\bullet$ becomes a box containing a $\bullet$, while simultaneously every box with a $\bullet$ and a $\mathcal{G}$ neighbor becomes a box of $\mathcal{G}$. The remaining boxes are unchanged by $\operatorname{switch}_{\mathcal{G}}$.

Index the genes of $T$ as

$$
\mathcal{G}_{1}<\mathcal{G}_{2}<\cdots<\mathcal{G}_{|\mu|}
$$

according to the total order on genes from Lemma 2.1. Then

$$
\mathrm{jdt}_{I}(T):=\operatorname{switch}_{\mathcal{G}_{|\mu|}}^{\bullet} \cdots \circ \operatorname{switch}_{\mathcal{G}_{2}}^{\bullet} \circ \operatorname{switch}_{\mathcal{G}_{1}}\left(T^{\bullet}\right)
$$

with the $\bullet$ 's deleted. (This algorithm reduces to M.-P. Schützenberger's jeu de taquin for semistandard tableaux in the case each gene contains only a single box.) 
Example 4.1. Suppose $T^{\bullet}$ is the genomic tableau \begin{tabular}{|l|l|l|l|}
\hline & & & $\bullet$ \\
\hline & $\bullet$ & 1 & 2 \\
\hline 1 & 1 & 2 & \\
\hline 2 &
\end{tabular}

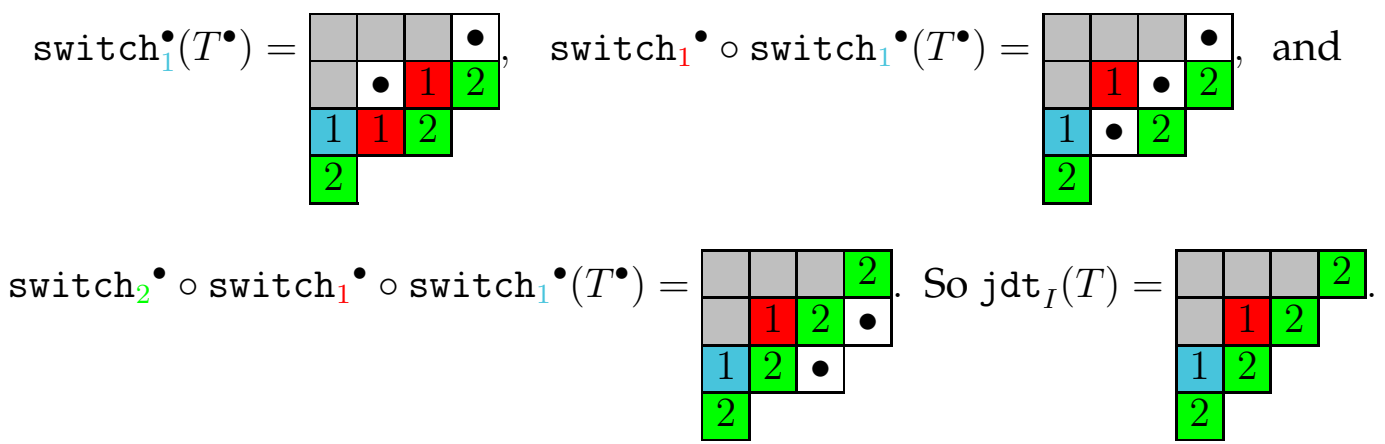

Define jeu de taquin equivalence $\sim_{G}$ on genomic tableaux as the symmetric, transitive closure of the relation $T \sim_{G} \mathrm{jdt}_{I}(T)$. We now state the genomic analogue of [BuSa13, Theorem 6.2] (restated as Theorem 4.3 below):

Theorem 4.2. Let $T, U$ be genomic tableaux. Then $T \sim_{G} U$ if and only if genomicseq $(T) \equiv_{G}$ genomicseq $(U)$.

Proof. We assume the terminology and results on the Kjdt (jeu de taquin) for increasing tableaux of [ThYo09b, $\S 1]$ (see specifically pages 123-124). K-Knuth equivalence [BuSa13, §5] is the the symmetric, transitive closure of the following $K$-Knuth relations (our conventions are reversed from those of [BuSa13]; this has no effect on the applicability of their results): For words $\mathbf{u}, \mathbf{v}$ and integers $0<i<j<k$,

$$
\begin{aligned}
\mathbf{u} i i \mathbf{v} & \equiv_{K} \mathbf{u} i \mathbf{v} \\
\mathbf{u} i j i \mathbf{v} & \equiv_{K} \mathbf{u} j i j \mathbf{v}, \\
\mathbf{u} j i k \mathbf{v} & \equiv_{K} \mathbf{u} j k i \mathbf{v}, \\
\mathbf{u} i k j \mathbf{v} & \equiv_{K} \mathbf{u} k i j \mathbf{v} .
\end{aligned}
$$

Define Kjdt-equivalence $\left(\sim_{K}\right)$ on increasing tableaux as the symmetric, transitive closure of the relation $T \sim_{K} \operatorname{Kjdt}_{I}(T)$. The key relationship between these two equivalence relations is:

Theorem 4.3. [BuSa13, Theorem 6.2] $T \sim_{K} U$ if and only if $\operatorname{seq}(T) \equiv_{K} \operatorname{seq}(U)$.

Let $\operatorname{Gen}(\nu / \lambda)$ be the set of all genomic tableaux of shape $\nu / \lambda$.

Lemma 4.4. For $T \in \operatorname{Gen}(\nu / \lambda)$ and set of inner corners $I, \Phi\left(\operatorname{jdt}_{I}(T)\right)=\operatorname{Kjdt}_{I}(\Phi(T))$.

Proof. From the definitions, this is an easy induction on the number of genes of $T$.

Lemma 4.5. For any genomic words $\mathbf{u}$ and $\mathbf{v}$, we have $\mathbf{u} \equiv_{G} \mathbf{v}$ if and only if $\Phi(\mathbf{u}) \equiv_{K} \Phi(\mathbf{v})$.

Proof. Immediate from the definitions of $\equiv_{K}$ and $\equiv_{G}$. 
By Lemma 4.4, $T \sim_{G} U$ if and only if $\Phi(T) \sim_{K} \Phi(U)$. By Theorem 4.3, the latter relation is equivalent to

$$
\operatorname{seq}(\Phi(T)) \equiv_{K} \operatorname{seq}(\Phi(U)) .
$$

By Lemma 4.5 and Lemma 3.3(III), we see that

$$
\operatorname{seq}(\Phi(T)) \equiv_{K} \operatorname{seq}(\Phi(U))
$$

is equivalent to

$$
\operatorname{genomicseq}(T) \equiv_{G} \operatorname{genomicseq}(U) \text {. }
$$

Corollary 4.6. If $T \sim_{G} U$, then $T$ is ballot if and only if $U$ is ballot.

Proof. By Theorem 4.2 and Theorem 3.5.

Let $T_{\mu}$ be the highest weight tableau of shape $\mu$, i.e., the semistandard tableau whose $i$-th row uses only the label $i$. Note $T_{\mu}$ may be also regarded as a genomic tableau in a unique manner. Let $S_{\mu}:=\Phi\left(T_{\mu}\right)$ be the row superstandard tableau of shape $\mu$ (this is the tableau whose first row has entries $1,2,3, \ldots, \mu_{1}$, and whose second row has entries $\mu_{1}+1, \mu_{2}+2, \ldots, \mu_{1}+\mu_{2}$ etc.).

Corollary 4.7 (of Lemma 4.4). For $T \in \operatorname{Gen}(\nu / \lambda), T \sim_{G} T_{\mu}$ if and only if $\Phi(T) \sim_{K} S_{\mu}$.

Proof. This is immediate from Lemma 4.4 because $S_{\mu}=\Phi\left(T_{\mu}\right)$.

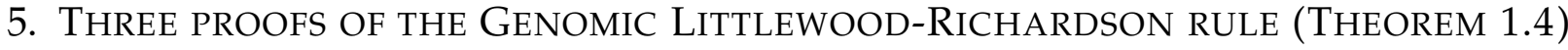

5.1. Proof 1: Bijection with increasing tableaux. Our first proof uses the results of Sections $2-4$ to prove Theorem 1.4 Let

$$
\operatorname{Ballot}_{\mu}(\nu / \lambda):=\left\{T \in \operatorname{Gen}_{\mu}(\nu / \lambda): T \text { is ballot }\right\} .
$$

Also, let

$$
\operatorname{IncRect}_{\mu}(\nu / \lambda):=\left\{T \in \operatorname{Inc}(\nu / \lambda): \operatorname{KRect}(T)=S_{\mu}\right\} .
$$

Lemma 5.1. Let $T \in \operatorname{Gen}_{\mu}(\nu / \lambda)$. Then $T \in \operatorname{Ballot}_{\mu}(\nu / \lambda)$ if and only if $\Phi(T) \in \operatorname{IncRect}_{\mu}(\nu / \lambda)$.

Proof. Suppose $T$ is ballot. By iterating application of $j \mathrm{dt} \mathrm{t}_{I}$ (under arbitrary choices of nonempty sets $I$ of inner corners) starting with $T$, we have that $T \sim_{G} R$ for some straightshaped tableau $R$ (a priori, $R$ might depend on the choices of $I$ ). By Corollary $4.6, R$ is ballot. Since genomic jeu de taquin preserves tableau content, $R=T_{\mu}$. Hence, by Lemma 4.4 , $\Phi(T)$ rectifies to $S_{\mu}$.

Conversely, suppose $\Phi(T)$ rectifies to $S_{\mu}$. Then by Lemma 4.4, $T$ rectifies to $T_{\mu}$. But $T_{\mu}$ is a ballot genomic tableau. Hence by Corollary $4.6, T$ is also ballot.

Lemma 5.2. $\operatorname{IncRect}_{\mu}(\nu / \lambda) \subseteq \mathrm{PF}_{\mu}(\nu / \lambda)$.

Proof. This is part of [ThYo09b, Proof of Theorem 1.2]. 
In view of Lemmas 5.1 and 5.2, we may define

$$
\phi: \operatorname{Ballot}_{\mu}(\nu / \lambda) \rightarrow \operatorname{IncRect}_{\mu}(\nu / \lambda)
$$

as the restriction

$$
\left.\Phi\right|_{\operatorname{Ballot}_{\mu}(\nu / \lambda)}
$$

and define

as the restriction

$$
\psi: \operatorname{IncRect}_{\mu}(\nu / \lambda) \rightarrow \operatorname{Ballot}_{\mu}(\nu / \lambda)
$$

$$
\left.\Psi\right|_{\text {IncRect }_{\mu}(\nu / \lambda)} .
$$

Now $\Phi$ and $\Psi$ are mutually inverse bijections (cf. Theorem 2.4). Thus $\phi$ and $\psi$ are mutually inverse bijections between $\operatorname{IncRect}_{\mu}(\nu / \lambda)$ and $\operatorname{Ballot}_{\mu}(\nu / \lambda)$. Hence the theorem follows from the Kjdt rule of [ThYo09b] for $a_{\lambda, \mu}^{\nu}$.

5.2. Proof 2: Bijection with set-valued tableaux. In our next proof, we relate genomic tableaux to the original rule for $a_{\lambda, \mu}^{\nu}$ found by A. Buch [Bu02, Theorem 5.4].

We first recall some definitions from [Bu02]. A set-valued tableau $T$ of (skew) shape $\nu / \lambda$ is a filling of the boxes of $\nu / \lambda$ with non-empty finite subsets of $\mathbb{N}$ with the property that any tableau obtained by choosing exactly one label from each box is a (classical) semistandard tableau. The column reading word of $T$, denoted $\operatorname{colword}(T)$ is obtained by reading the entries of $T$ from bottom to top along columns and from left to right. The entries in a non-singleton box are read in increasing order. Such a word $\left(w_{1}, w_{2}, \ldots, w_{N}\right)$ is a reverse lattice word if the content of $\left(w_{L}, w_{L+1}, \ldots, w_{N}\right)$ is a partition for every $1 \leq L \leq$ $N$, that is to say if its reverse is ballot. Finally, the shape $\mu \star \lambda$ is the skew shape obtained by placing $\mu$ and $\lambda$ in southwest to northeast orientation with $\mu^{\prime}$ s northeast corner incident to $\lambda^{\prime}$ s southwest corner. In other words

$$
\mu \star \lambda=\left(\mu_{1}+\lambda_{1}, \ldots, \mu_{1}+\lambda_{\ell(\lambda)}, \mu_{1}, \mu_{2}, \ldots, \mu_{\ell(\mu)}\right) /\left(\mu_{1}^{\ell(\lambda)}\right) .
$$

Example 5.3. If $\lambda=\boxplus$ and $\mu=\boxplus$, then $\mu \star \lambda={ }^{\top} \boxplus$.

Theorem 5.4 (A. Buch [Bu02, Theorem 5.4]). $(-1)^{|\lambda|+|\mu|-|\nu|} a_{\lambda, \mu}^{\nu}$ equals the number of setvalued tableaux $T$ of shape $\mu \star \lambda$ and content $\nu$ such that $\operatorname{colword}(T)$ is reverse lattice.

Let $\operatorname{Buch}_{\nu}(\mu \star \lambda)$ be the set of tableaux from Theorem 5.4. We define a map

$$
\Xi: \operatorname{Buch}_{\nu}(\mu \star \lambda) \rightarrow \operatorname{Ballot}_{\mu}(\nu / \lambda)
$$

as follows. Let $T \in \operatorname{Buch}_{\nu}(\mu \star \lambda)$. Start with an empty shape $\lambda$. Read the columns of the $\mu$ portion of $T$ from top to bottom and right to left. Suppose a set

$$
S=\left\{s_{1}<\ldots<s_{t}\right\}
$$

gives the entries of a box in row $i$ of the $\mu$ shape. Then place a new gene of family $i$ in the rows $s_{1}, \ldots, s_{t}$ (as far left as possible in each case). Then $\Xi$ clearly has a (putative) inverse

$$
\Theta: \operatorname{Ballot}_{\mu}(\nu / \lambda) \rightarrow \operatorname{Buch}_{\nu}(\mu \star \lambda)
$$

that records in row $i$ and column 1 of the $\mu$ shape the rows that the leftmost gene of family $i$ sits in. Similarly, in row $i$ and column 2 we record the rows that the second leftmost gene of family $i$ sits in, etc.

Theorem 5.5. $\Xi: \operatorname{Buch}_{\nu}(\mu \star \lambda) \rightarrow \operatorname{Ballot}_{\mu}(\nu / \lambda)$ and $\Theta: \operatorname{Ballot}_{\mu}(\nu / \lambda) \rightarrow \operatorname{Buch}_{\nu}(\mu \star \lambda)$ are well-defined and mutually inverse bijections. 
Example 5.6. Let $\lambda=(2,1), \mu=(1,1)$ and $\nu=(3,2,1)$. Then $\operatorname{Buch}_{\nu}(\mu \star \lambda)$ consists of the two tableaux

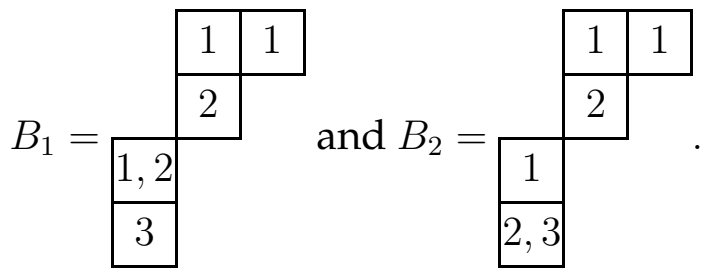

We have

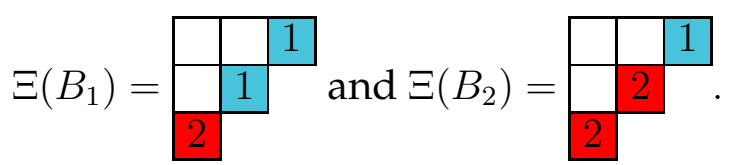

The reader can check that these are the unique two elements of $\operatorname{Ballot}_{\mu}(\nu / \lambda)$.

Proof of Theorem 5.5. Let $T \in \operatorname{Buch}_{\nu}(\mu \star \lambda)$ and set $U:=\Xi(T)$.

( $\Xi$ is well-defined): By definition, the number of genes of family $i$ is $\mu_{i}$. Hence the content of $U$ is $\mu$, as required. Next, observe that since in each row of $T$ the entries increase weakly from left to right, no two genes of the same family interweave. Also note that no two labels of the same gene are in the same column since otherwise we would obtain that $\operatorname{colword}(T)$ is not reverse lattice, since labels in the same box are read in increasing order, a contradiction.

The hypothesis that colword $(T)$ is reverse lattice precisely guarantees that when adding the boxes in the rows of $S$ one takes a Young diagram to a larger Young diagram. Thus $U$ is a tableau of (skew) Young diagram shape. Note that since $S$ is a set, no row of $U$ contains two boxes of the same gene.

We next verify the semistandardness conditions. Suppose $U$ violates the horizontal semistandardness requirement. That is, there is a box $x$ directly left and adjacent to a box $y$ in $U$ such that $\operatorname{lab}_{U}(x)>\operatorname{lab}_{U}(y)$. Let $x^{\prime}$ and $y^{\prime}$ be the boxes in $T$ that added $x$ and $y$ during the execution of $\Xi$. Since $\operatorname{lab}_{U}(x)>1 \mathrm{ab}_{U}(\mathrm{y})$, by $\Xi^{\prime}$ s definition, the row of $x^{\prime}$ is strictly south of the row of $y^{\prime}$. Moreover, since $x$ is left of $y$ we know that $x^{\prime}$ is read before $y^{\prime}$ in $\operatorname{col} \operatorname{word}(T)$. Therefore, $y^{\prime}$ is strictly north and strictly west of $x^{\prime}$. However, since $T$ is a (set-valued) semistandard tableau, the labels of $y^{\prime}$ in $T$ are all strictly smaller than those of $x^{\prime}$. This implies that $y$ is in a row strictly north of that of $x$, a contradiction. The argument that $U$ satisfies the vertical semistandardness requirement is similar.

It remains to check that $U$ is ballot. To do this, make an arbitrary but fixed choice of genotype $G_{U}$ of $U$. The labels of family $i$ and $i+1$ may be blamed on labels in rows $i$ and $i+1$ of $T$. Suppose the sets of labels in those rows are

$$
Q_{1}, Q_{2}, \ldots, Q_{t}, Q_{t+1}, \ldots, Q_{t+s}(\text { row } i) \text { and } R_{1}, R_{2}, \ldots, R_{t}(\text { row } i+1)
$$

where $s \geq 0$. Since we know $U$ is semistandard, the labels associated to rows $i$ and $i+1$ separately form a Pieri strip. Here $Q_{1}$ is associated to the rightmost gene of family $i$ (in $U$ ) and $Q_{t+s}$ is associated to the leftmost gene of family $i$ (in $U$ ). Similarly, $R_{1}$ is associated to the rightmost gene of family $i+1$ (in $U$ ) and $R_{t}$ is associated to the leftmost gene of family $i+1$ (in $U$ ). By the vertical semistandardness of $T$, we have

$$
\max Q_{i}<\min R_{i} \text { for } 1 \leq i \leq t .
$$


This clearly implies that the $m$ th rightmost label of family $i+1$ in $G_{U}$ is strictly south and weakly west of the $m$ th rightmost label of family $i$ in $G_{U}$, for $1 \leq m \leq t$. Since this is true for each $i, G_{U}$ is ballot.

( $\Xi$ is injective): Clear.

( $\Theta$ is well-defined:) This is proved with the same arguments (said in reverse) as those given in the well-definedness of $\Xi$.

$(\Theta$ is injective): Clear.

The theorem follows since $\Xi$ and $\Theta$ are mutually inverse injections.

Composing Theorem [5.5] with the bijection of Section 5.1permits one to biject the above rule of A. Buch with the $K$-theoretic jeu de taquin rule of [ThYo09b].

5.3. Proof 3: Bijection with puzzles. A third proof of Theorem 1.4 considers the bijection given in [PeYo15c] between more general genomic tableaux and the Knutson-Vakil puzzles of [CoVa05, §5]. It is not hard to see that this restricts to a bijection between the tableaux of Theorem 1.4 and the ordinary $K$-theory puzzles of A. Buch [Va06, §3.3]. Since the latter are known to calculate $a_{\lambda, \mu}^{\nu}$, Theorem 1.4 follows.

Consider the $n$-length equilateral triangle oriented as $\Delta$. A puzzle is a filling of $\Delta$ with the following puzzle pieces:
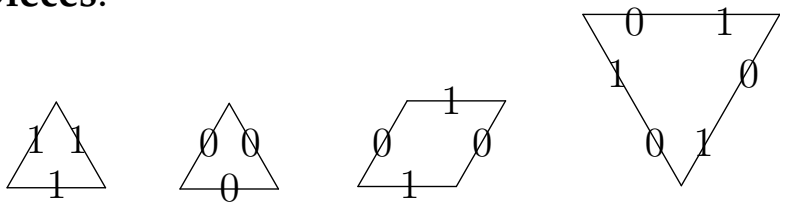

Henceforth, we color code these pieces as black, white, gray, and blue respectively, dropping the numerical labels. A filling requires that the common edges of adjacent puzzle pieces share the same label. The first three may be rotated but the fourth ( $K$-piece) may not. A $K$-puzzle is a puzzle filling of $\Delta$.

Convert partitions inside a $k \times(n-k)$ rectangle to binary sequences in the following way. Starting at the upper right corner of the $k \times(n-k)$ rectangle, construct a lattice path from the binary sequence by reading each segment $(-1,0)$ as 0 and each segment $(0,-1)$ as 1 . For example, we convert the partition $(3,2)$ to the binary string 010100 as follows:

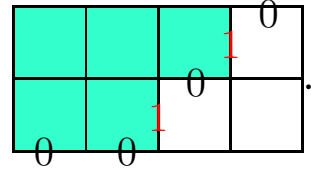

Let $\Delta_{\lambda, \mu, \nu}$ be $\Delta$ with the boundary given by binary sequences

- $\lambda$ as read $\nearrow$ along the left side;

- $\mu$ as read $\searrow$ along the right side; and

- $\nu$ as read $\rightarrow$ along the bottom side.

Theorem 5.7 (A. Buch [Va06, §3.3]). $(-1)^{|\nu|-|\lambda|-|\mu|} a_{\lambda, \mu}^{\nu}=\#\left\{\right.$ K-puzzles of $\left.\Delta_{\lambda, \mu, \nu}\right\}$. 
Example 5.8. Continuing Example 5.6 and assuming the Grassmannian in question is $\mathrm{Gr}_{3}\left(\mathbb{C}^{6}\right)$, the bijection of [PeYo15c] matches $\Xi\left(B_{1}\right)$ and $\Xi\left(B_{2}\right)$ to the puzzles
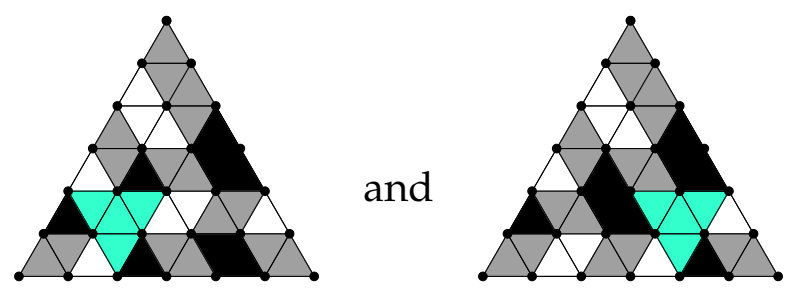

respectively, where we use the color-coding of puzzle pieces described above. It is straightforward to check that these are the only $K$-puzzles in the sense of [Va06, §3.3] for this structure constant.

\section{INFUSION, BENDER-KNUTH INVOLUTIONS AND THE GENOMIC SCHUR FUNCTION}

We first define genomic infusion. Let $T \in \operatorname{Gen}(\alpha)$ and $U \in \operatorname{Gen}(\beta / \alpha)$ where $\alpha$ is possibly a skew shape. We think of a layered tableau $(T, U)$ that is the union of $T$ and $U$. For convenience, the labels of $T$ will be circled. Then

$$
\text { geninf }(T, U)=\left(U^{\star}, T^{\star}\right)
$$

is obtained by the following procedure. Consider the largest gene $(\mathcal{G}$ (under the $<$ order) that appears in $T$. The boxes of this gene are inner corners $I$ with respect to $U$. Now apply jdt ${ }_{I}(U)$, leaving some outer corners of $\beta$. Place into these outer corners (G). Now consider the second largest gene $\mathcal{G}^{\prime}$ that appears in $T$. These will form inner corners $I^{\prime}$ with respect to $U^{\prime}:=j \mathrm{dt}_{I}(U)$. Now apply $\mathrm{jdt} \mathrm{I}_{I^{\prime}}\left(U^{\prime}\right)$ again leaving some outer corners of which we will fill with $\mathcal{G}^{\prime}$. We continue in this manner until we have exhausted all genes of $T$. The "inner" tableau of uncircled genes is $U^{\star}$ and the "outer" tableau of circled genes is $T^{\star}$. Clearly, if $\alpha$ is a straight shape, then $U^{\star}$ is a genomic rectification of $U$ where the order of rectification is imposed by $T$. Furthermore:

Proposition 6.1. Genomic infusion is an involution, i.e.,

$$
\operatorname{geninf}\left(U^{\star}, T^{\star}\right)=(T, U) .
$$

Proof. This follows from the fact that K-infusion as defined in [ThYo09b, §3.1] is an involution [ThYo09b, Theorem 3.1], combined with Lemma 4.4.

Next we define genomic Bender-Knuth involutions. Given a genomic tableau $V$ consider the genomic subtableau $T$ consisting of genes of family $i$ and consider the genomic subtableau $U$ consisting of genes of family $i+1$. Now define genBK ${ }_{i}(V)$ to be obtained by replacing inside $V$ the subtableau $(T, U)$ with $\left(U^{\star}, T^{\star}\right)$, switching the labels $i$ and $i+1$, keeping all other boxes of $V$ the same (and removing any circlings).

Proposition 6.2. genBK ${ }_{i}$ is an involution. Moreover, genBK ${ }_{i}$ defines a bijection from the set of genomic tableaux of a shape $\nu / \lambda$ of content $\gamma=\left(\gamma_{1}, \ldots, \gamma_{i}, \gamma_{i+1}, \ldots\right)$ to the set of genomic tableaux of shape $\nu / \lambda$ of content $\gamma=\left(\gamma_{1}, \ldots, \gamma_{i+1}, \gamma_{i}, \ldots\right)$.

Proof. The first sentence is immediate from Proposition 6.1. The second sentence follows from the definition of genBK ${ }_{i}$ and the first sentence. 
From these genomic Bender-Knuth involutions, one can define genomic versions of M.-P. Schützenberger's promotion and evacuation operators. (For the classical theory, see [BlPeSa16], specifically Theorems 2.2 and 2.9, as well as the references therein.) We do not analyze these notions further in this paper.

We explore the genomic Schur function, which we define as

$$
U_{\nu / \lambda}:=\sum_{T \in \operatorname{Gen}(\nu / \lambda)} \mathbf{x}^{T}
$$

where

$$
\mathbf{x}^{T}:=\prod_{i} x_{i}^{\# \text { genes of family } i \text { in } T} .
$$

Example 6.3. The polynomial $U_{31}\left(x_{1}, x_{2}\right)$ is computed by the tableaux

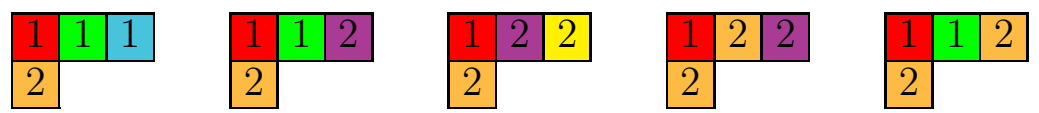

Hence $U_{31}\left(x_{1}, x_{2}\right)=x_{1}^{3} x_{2}+x_{1}^{2} x_{2}^{2}+x_{1} x_{2}^{3}+x_{1} x_{2}^{2}+x_{1}^{2} x_{2}=s_{31}\left(x_{1}, x_{2}\right)+s_{21}\left(x_{1}, x_{2}\right)$.

Theorem 6.4. $U_{\nu / \lambda} \in$ Sym.

Proof. The argument is an extension of the combinatorial proof of symmetry of Schur functions: It follows from Proposition 6.2.

Since

$$
U_{\nu / \lambda}=s_{\nu / \lambda}+\text { lower degree terms, }
$$

by Theorem 6.4 we have that $\left\{U_{\lambda}\right\}$, where $\lambda$ ranges over all (straight) partitions, is a basis of Sym.

While in small examples $U_{\nu / \lambda}$ is Schur-positive (cf. Table1), this is not true in general:

Example 6.5. One may check that 38 tableaux contribute to $U_{333}\left(x_{1}, x_{2}, x_{3}, x_{4}\right)$. Expanding this polynomial in the Schur basis yields

$$
\begin{aligned}
U_{333}\left(x_{1}, x_{2}, x_{3}, x_{4}\right)=s_{333}\left(x_{1}, x_{2}, x_{3}, x_{4}\right) & +s_{3221}\left(x_{1}, x_{2}, x_{3}, x_{4}\right) \\
& +s_{2221}\left(x_{1}, x_{2}, x_{3}, x_{4}\right)-s_{2222}\left(x_{1}, x_{2}, x_{3}, x_{4}\right) .
\end{aligned}
$$

Also, the structure coefficients for the $U$-basis do not possess any positivity or alternating positivity properties:

Example 6.6. Using Table 1, one can check that $U_{22} \cdot U_{1}=U_{32}+U_{221}-U_{22}-U_{111}$.

At present, we are unaware of any geometric significance of these polynomials.

\section{SHIFTED GENOMIC TABLEAUX}

Recall, the shifted diagram of a strictly decreasing partition is given by taking the ordinary Young diagram and indenting row $i$ (from the top) $i-1$ positions to the right. Let

$$
\mathcal{D}:=\left\{1^{\prime}<1<2^{\prime}<2<\cdots\right\} \text {. }
$$

A $P$-tableau is a filling of shifted shape $\nu / \lambda$ with entries from $\mathcal{D}$ such that:

(P.1) rows and columns weakly increase (left to right, top to bottom); 


\begin{tabular}{|c|c|c|c|c|c|c|c|c|c|c|c|c|c|c|c|c|c|c|c|}
\hline$\lambda \backslash \mu$ & $\square$ & ㅁ & 日 & मा & $\Phi$ & 日 & 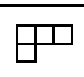 & 田 & 甲 & $\boxplus$ & 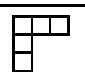 & $\bigoplus$ & $\boxplus$ & $\bigoplus$ & $\boxplus$ & \# & $\boxplus$ & 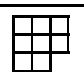 & 曲 \\
\hline$\square$ & 1 & 0 & 0 & 0 & 0 & 0 & 0 & 0 & 0 & 0 & 0 & 0 & 0 & 0 & 0 & 0 & 0 & 0 & 0 \\
\hline$\square$ & 0 & 1 & 0 & 0 & 0 & 0 & 0 & 0 & 0 & 0 & 0 & 0 & 0 & 0 & 0 & 0 & 0 & 0 & 0 \\
\hline 日 & 0 & 0 & 1 & 0 & 0 & 0 & 0 & 0 & 0 & 0 & 0 & 0 & 0 & 0 & 0 & 0 & 0 & 0 & 0 \\
\hline पाप & 0 & 0 & 0 & 1 & 0 & 0 & 0 & 0 & 0 & 0 & 0 & 0 & 0 & 0 & 0 & 0 & 0 & 0 & 0 \\
\hline 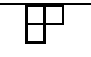 & 0 & 0 & 1 & 0 & 1 & 0 & 0 & 0 & 0 & 0 & 0 & 0 & 0 & 0 & 0 & 0 & 0 & 0 & 0 \\
\hline 日 & 0 & 0 & 0 & 0 & 0 & 1 & 0 & 0 & 0 & 0 & 0 & 0 & 0 & 0 & 0 & 0 & 0 & 0 & 0 \\
\hline$\nabla^{\square}$ & 0 & 0 & 0 & 0 & 1 & 0 & 1 & 0 & 0 & 0 & 0 & 0 & 0 & 0 & 0 & 0 & 0 & 0 & 0 \\
\hline$\boxplus$ & 0 & 0 & 0 & 0 & 0 & 1 & 0 & 1 & 0 & 0 & 0 & 0 & 0 & 0 & 0 & 0 & 0 & 0 & 0 \\
\hline 由 & 0 & 0 & 0 & 0 & 0 & 2 & 0 & 0 & 1 & 0 & 0 & 0 & 0 & 0 & 0 & 0 & 0 & 0 & 0 \\
\hline$\boxplus$ & $\overline{0}$ & $\overline{0}$ & 0 & 0 & $\overline{0}$ & 1 & $\overline{0}$ & 1 & $\overline{1}$ & 1 & $\overline{0}$ & 0 & 0 & 0 & $\overline{0}$ & 0 & $\overline{0}$ & 0 & 0 \\
\hline$\theta^{1}$ & 0 & 0 & 0 & 0 & 0 & 1 & 0 & 0 & 2 & 0 & 1 & 0 & 0 & 0 & 0 & 0 & 0 & 0 & 0 \\
\hline$\boxplus$ & 0 & 0 & 0 & 0 & 0 & 1 & 0 & 0 & 1 & 0 & 0 & 1 & 0 & 0 & 0 & 0 & 0 & 0 & 0 \\
\hline$\boxplus$ & $\overline{0}$ & 0 & 0 & 0 & 0 & 0 & 0 & 0 & 0 & 0 & 0 & 1 & 1 & 0 & 0 & 0 & 0 & 0 & 0 \\
\hline$\boxplus$ & 0 & 0 & 0 & 0 & 0 & 1 & 0 & 0 & 2 & 0 & 1 & 2 & 0 & 1 & 0 & 0 & 0 & 0 & 0 \\
\hline$\boxplus$ & 0 & 0 & 0 & 0 & 0 & 0 & 0 & 0 & 0 & 0 & 0 & 0 & 0 & 0 & 1 & 0 & 0 & 0 & 0 \\
\hline 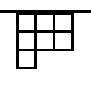 & 0 & 0 & 0 & 0 & 0 & 0 & 0 & 0 & 0 & 0 & 0 & 1 & 0 & 1 & 0 & 1 & 0 & 0 & 0 \\
\hline$\boxplus$ & 0 & 0 & 0 & 0 & 0 & 0 & 0 & 0 & 0 & 0 & 0 & 0 & 0 & 0 & 2 & 0 & 1 & 0 & 0 \\
\hline$\boxplus$ & 0 & 0 & 0 & 0 & 0 & 0 & 0 & 0 & 0 & 0 & 0 & 0 & 0 & 0 & 1 & 0 & 1 & 1 & 0 \\
\hline 曲 & 0 & 0 & 0 & 0 & 0 & 0 & 0 & 0 & 0 & 0 & 0 & 0 & 0 & 0 & 0 & 0 & 0 & 0 & 1 \\
\hline
\end{tabular}

TABLE 1. Transition matrix between the $\left\{U_{\lambda}\left(x_{1}, x_{2}, x_{3}\right)\right\}$ to $\left\{s_{\mu}\left(x_{1}, x_{2}, x_{3}\right)\right\}$ bases.

(P.2) each unprimed letter appears at most once in any column;

(P.3) each primed letter appears at most once in any row; and

(P.4) every primed letter $k^{\prime}$ has an unprimed $k$ southwest of it.

The Schur P-function $P_{\lambda}$ is a generating function over these tableaux (for more history and development of these functions, see e.g., [HoHu92] or[St89]).

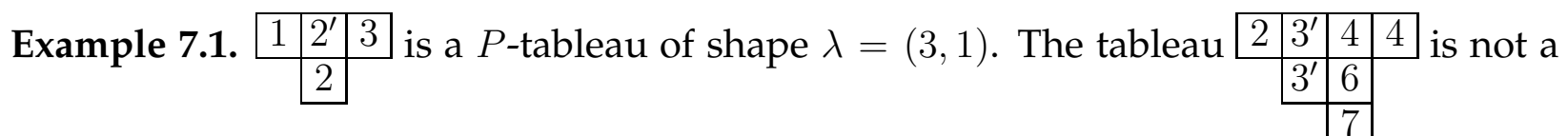
$P$-tableau because it violates both (P.3) and (P.4). However, if the lower $3^{\prime}$ changes to 3, the result is a $P$-tableau.

For $\alpha \in \mathcal{D}$, write $|\alpha|=k$ if $\alpha \in\left\{k^{\prime}, k\right\}$. We use initial letters of the Greek alphabet $(\alpha, \beta, \gamma, \ldots)$ for elements of $\mathcal{D}$, reserving Roman letters for elements of $\mathbb{Z}$. 
For fixed $k \in \mathbb{Z}_{\geq 0}$, place a total order $\prec$ on those boxes with entry $k^{\prime}$ in top to bottom order and on those boxes with entry $k$ using left to right order; declare the boxes containing $k^{\prime}$ to precede those containing $k$. A gene (of family $k$ ) in a $P$-tableau $T$ is a set $\mathcal{G}$ of boxes of $T$ such that:

- each entry in $\mathcal{G}$ is $k^{\prime}$ or $k$;

- the boxes of $\mathcal{G}$ are consecutive in the $\prec$-order; and

- no two boxes of $\mathcal{G}$ appear in the same row or the same column.

We write $\operatorname{family}(\mathcal{G})=k$.

Example 7.2. Consider the following three colorings of the same $P$-tableau:
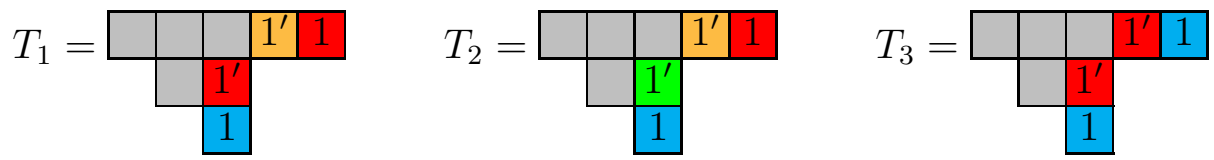

The red boxes in $T_{1}$ do not form a gene, since they are not consecutive in $\prec$-order (in view of the blue 1). In $T_{2}$ and $T_{3}$, the boxes of each color form valid genes.

A genomic $P$-tableau is a $P$-tableau $T$ together with a partition of its boxes into genes such that for every primed box $b$, there is an box $c$ that is weakly southwest of $b$ from $a$ different gene than $b$ but of the same family. The content of $T$ is the number of genes of each family. A genotype $G$ of $T$ is a choice of a single box from each gene. Depict $G$ by erasing the entries in boxes that are not chosen. A $P$-tableau may be identified with the genomic $P$-tableau where each box is its own gene.

Example 7.3. Let $\nu=(6,4,1)$ and $\lambda=(4,2)$. Then a genomic $P$-tableau $T$ of shape $\nu / \lambda$ and its two genotypes $G_{1}, G_{2}$ are
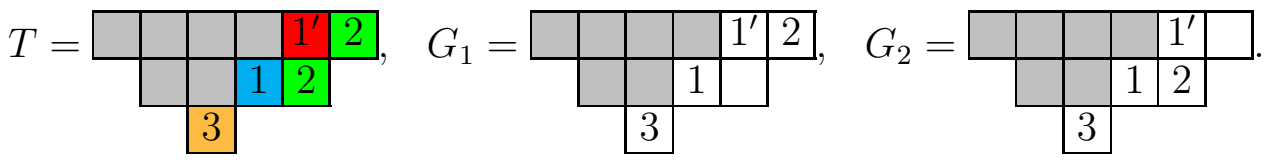

The content of $T$ is $\mu=(2,1,1)$.

Given a word $w$ using the alphabet $\mathcal{D}, \hat{w}$ is the word obtained by writing $w$ backwards, and replacing each $k^{\prime}$ with $k$ while simultaneously replacing each $k$ with $(k+1)^{\prime}$. Let

$$
\text { doubleseq }(G):=\operatorname{seq}(G) \widehat{\operatorname{seq}(G)} .
$$

Say doubleseq $(G)$ is locally ballot at the letter $\alpha \in \mathcal{D}$, if $|\alpha|=1$ or if in doubleseq $(G)$ the number of $|\alpha|$ 's appearing strictly before that $\alpha$ is strictly less than the number of $(|\alpha|-1)$ 's appearing strictly before that $\alpha$. Declare doubleseq $(G)$ to be ballot if it is locally ballot at each letter. Finally, $G$ is ballot if doubleseq $(G)$ is ballot, and the genomic $P$-tableau $T$ is ballot if every genotype of $T$ is ballot.

Example 7.4. Let $G_{1}$ and $G_{2}$ be as in Example7.3, Then

$$
\text { doubleseq }\left(G_{1}\right)=21^{\prime} 134^{\prime} 2^{\prime} 13^{\prime} \text { and doubleseq }\left(G_{2}\right)=1^{\prime} 2134^{\prime} 2^{\prime} 3^{\prime} 1 \text {. }
$$

The former is not ballot, as it starts with 2. Hence the genomic $P$-tableau $T$ of Example7.3 is not ballot. $G_{2}$ is also not ballot: doubleseq $\left(G_{2}\right)$ is locally ballot at every position except the 2 in second position; although there is a $1^{\prime}$ before this 2 , there is no 1 . To emphasize the differences between ballotness in this section versus ballotness in Section 3, note that deleting the primes gives 12134231, which is ballot in the earlier sense. 
A $Q$-tableau is a filling of $\nu / \lambda$ with entries from $\mathcal{D}$ satisfying (P.1)-(P.3) and

(Q.4) no primed letters appear on the main diagonal.

(Observe that (Q.4) is a weakening of (P.4), so a $P$-tableau is a $Q$-tableau.)

A gene (of family $k$ ) in a $Q$-tableau $T$ is a set $\mathcal{G}$ of boxes such that:

- each entry of $\mathcal{G}$ is $k^{\prime}$ or $k$,

- the boxes of $\mathcal{G}$ are consecutive in the $\prec$-order, and

- no two boxes of $\mathcal{G}$ with the same label appear in the same row or the same column.

We write $\operatorname{family}(\mathcal{G})=k$ as before.

A genomic $Q$-tableau is a $Q$-tableau $T$ together with a partition of its boxes into genes. The definition of ballotness for genomic $Q$-tableaux is the same as for genomic $P$-tableaux. Let $\mathrm{PGen}_{\mu}(\nu / \lambda)$ and $\mathrm{QGen}_{\mu}(\nu / \lambda)$ respectively denote the sets of genomic $P$ - and $Q$-tableaux of shape $\nu / \lambda$ and content $\mu$.

Lemma 7.5. $\operatorname{PGen}_{\mu}(\nu / \lambda) \subseteq \mathrm{QGen}_{\mu}(\nu / \lambda)$.

Proof. Let $T \in \operatorname{PGen}_{\mu}(\nu / \lambda)$. The definition of a gene in a $Q$-tableau differs from that for $P$-tableaux only in that it allows $k^{\prime}$ and $k$ in the same row or column to be in the same gene. Hence each gene of $T$ is a gene in the $Q$-tableau sense. Thus $T \in \mathrm{QGen}_{\mu}(\nu / \lambda)$.

In the announcement version of this paper ([PeYo15a, §3.2]), we used tableaux of selfconjugate shape rather than shifted shape. This is equivalent to our present discussion, as those tableaux may be recovered by reflecting the shifted tableaux.

\section{MAXIMAL ORTHOGONAL AND LAGRANGIAN GRASSMANNIANS}

Let $G / P$ be a generalized flag variety, where $G$ is a complex, connected, reductive Lie group and $P$ is a parabolic subgroup containing a Borel subgroup $B$. Let $B_{-}$be the opposite Borel to $B$ with respect to a choice of maximal torus $T \subseteq B$. The Schubert cells of $G / P$ are the $\mathbf{B}_{-}$-orbits, and the Schubert varieties $V_{\lambda}$ are their closures. Here $\lambda \in W / W_{\mathbf{P}}$ where $W$ is the Weyl group of $\mathrm{G}$ and $W_{\mathrm{P}}$ is the parabolic subgroup of $W$ corresponding to $\mathrm{P}$. The classes of Schubert structure sheaves $\left\{\left[\mathcal{O}_{V_{\lambda}}\right]\right\}$ form a $\mathbb{Z}$-linear basis of the Grothendieck ring $K^{0}(\mathrm{G} / \mathrm{P})$. Let $t_{\lambda, \mu}^{\nu}$ be the structure constants with respect to this basis. A. Buch [Bu02, Conjecture 9.2] conjectured the sign-alternation:

$$
(-1)^{\operatorname{codim}_{\mathrm{G} / \mathrm{P}}\left(V_{\nu}\right)-\operatorname{codim}_{\mathrm{G} / \mathrm{P}}\left(V_{\lambda}\right)-\operatorname{codim}_{\mathrm{G} / \mathrm{P}}\left(V_{\mu}\right)} t_{\lambda, \mu}^{\nu} \geq 0 .
$$

This was subsequently proved by $\mathrm{M}$. Brion [Br02]. While the Grassmannian $X$ is the most well-studied case of $G / P$, we now turn to an investigation of the next two most important cases when $P$ is maximal parabolic.

Fix a non-degenerate, symmetric bilinear form $\beta(\cdot, \cdot)$ on $\mathbb{C}^{2 n+1}$. A subspace $V \subseteq \mathbb{C}^{2 n+1}$ is isotropic with respect to $\beta$ if $\beta(\vec{v}, \vec{w})=0$ for all $\vec{v}, \vec{w} \in V$. Let

$$
Y=\mathrm{OG}(n, 2 n+1)
$$

be the maximal orthogonal Grassmannian, i.e., the parameter space of all such isotropic $n$-dimensional subspaces in $\mathbb{C}^{2 n+1}$. Define the shifted staircase $\delta_{n}$ to be the shifted shape 
whose $i$ th row is of length $i$ for $1 \leq i \leq n$. The Schubert varieties $Y_{\lambda}$ of $Y$ are indexed by shifted Young diagrams

$$
\lambda=\left(\lambda_{1}>\lambda_{2}>\cdots>\lambda_{n}\right)
$$

contained in $\delta_{n}$, i.e.,

$$
\lambda_{k} \leq n-k+1 \text { for } 1 \leq k \leq n .
$$

We have

$$
\operatorname{codim}_{Y}\left(Y_{\lambda}\right)=|\lambda| .
$$

Let $b_{\lambda, \mu}^{\nu}$ be $t_{\lambda, \mu}^{\nu}$ in this case. The first combinatorial rule for $b_{\lambda, \mu}^{\nu}$ was conjectured in [ThYo09b] and proved in [ClThYo14], using [BuRa12].

The following is a new rule for these structure coefficients. This rule directly extends the rule of J. Stembridge [St89, Theorem 8.3] for the ordinary cohomological structure constants of $Y$. (J. Stembridge's rule is stated in terms of projective representation theory of symmetric groups; the application to $H^{\star}(Y)$ is due to P. Pragacz [Pr89].)

Theorem 8.1 (OG Genomic Littlewood-Richardson rule).

$b_{\lambda, \mu}^{\nu}=(-1)^{|\nu|-|\lambda|-|\mu|}$ times the number of ballot genomic P-tableaux of shape $\nu / \lambda$ with content $\mu$.

Example 8.2. (cf. [ClThYo14, Example 1.3]) That

$$
b_{(3,1),(3,1)}^{(5,3,1)}(\mathrm{OG}(n, 2 n+1))=-6
$$

is witnessed by:
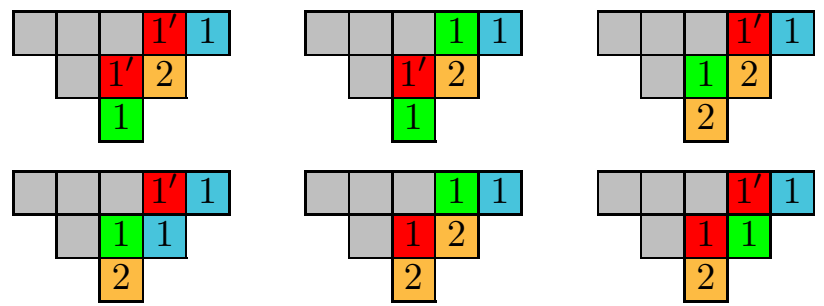

Fix a symplectic bilinear form $\omega(\cdot, \cdot)$ on $\mathbb{C}^{2 n}$. The Lagrangian Grassmannian

$$
Z=\mathrm{LG}(n, 2 n)
$$

is the parameter space of $n$-dimensional linear subspaces of $\mathbb{C}^{2 n}$ that are isotropic with respect to $\omega$. The Schubert varieties $\left\{Z_{\lambda}\right\}$ of $Z$ are indexed by the same shifted Young diagrams $\lambda$ as above; also, $\operatorname{codim}_{Z}\left(Z_{\lambda}\right)=|\lambda|$. Let $c_{\lambda, \mu}^{\nu}$ be $t_{\lambda, \mu}^{\nu}$ in this case.

There is a well-known relationship in the "cohomological case", i.e., when $|\lambda|+|\mu|=|\nu|$, between the structure constants for $Y$ and $Z$ :

$$
c_{\lambda, \mu}^{\nu}=2^{\ell(\lambda)+\ell(\mu)-\ell(\nu)} b_{\lambda, \mu}^{\nu},
$$

where $\ell(\pi)$ denotes the number of nonzero parts of $\pi$. We are not aware of any generalization of (2); cf. [BuRa12, Examples 4.9 and 5.8]. On the other hand, we propose the following extension of this relationship:

Conjecture 8.3. For any strict partitions $\lambda, \mu, \nu$, we have $\left|b_{\lambda, \mu}^{\nu}\right| \leq\left|c_{\lambda, \mu}^{\nu}\right|$. 
This conjecture is true in the cohomological case since it is known that $\ell(\lambda)+\ell(\mu) \geq \ell(\nu)$ whenever $b_{\lambda, \mu}^{\nu}>0$. Moreover, we have verified this conjecture by computer for $n \leq 6$. In addition, by [BuRa12], this conjecture holds whenever $\mu$ has a single part.

Let $\mathrm{QBallot} \mu(\nu / \lambda):=\{$ ballot genomic $Q$-tableaux of shape $\nu / \lambda$ with content $\mu\}$.

Conjecture 8.4. $\left|c_{\lambda, \mu}^{\nu}\right| \leq \# \mathrm{QBallot}_{\mu}(\nu / \lambda)$.

Example 8.5. Let $\lambda=(3,1), \mu=(2,1)$ and $\nu=(4,3,1)$. Then \#QBallot ${ }_{\mu}(\nu / \lambda)=6$ :

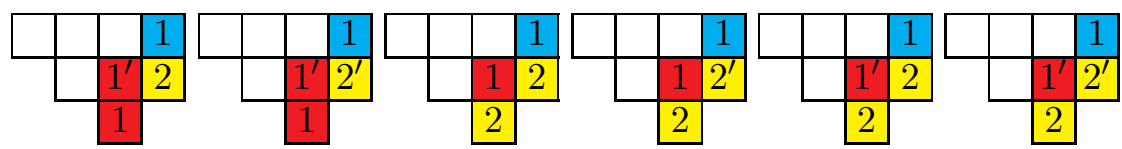

The third tableau above is the only one that is a genomic $P$-tableau; hence $b_{\lambda, \mu}^{\nu}=-1$. Therefore Conjectures 8.4 and 8.3 predict $1 \leq\left|c_{\lambda, \mu}^{\nu}\right| \leq 6$. Indeed, $c_{\lambda, \mu}^{\nu}=-5$.

We have computer verified Conjecture 8.4 for $n \leq 6$. Moreover, the bound is sharp, as indicated in the two propositions below.

Proposition 8.6. For $\mu=(p),\left|c_{\lambda, \mu}^{\nu}\right|=\# \mathrm{QBallot}{ }_{\mu}(\nu / \lambda)$.

Proof. By applying $\Gamma$ (defined in Section 9.1) to the tableaux in $\operatorname{QBallot}_{(p)}(\nu / \lambda)$ and retaining the primes, one obtains precisely the KLG-tableaux of A. Buch-V. Ravikumar [BuRa12, $\S 5]$. By [BuRa12, Corollary 5.6], the number of the latter is $(-1)^{|\nu|-|\lambda|-p} c_{\lambda,(p)}^{\nu}$.

Proposition 8.7. For $|\nu| \leq|\lambda|+|\mu|,\left|c_{\lambda, \mu}^{\nu}\right|=\# \mathrm{QBallot}_{\mu}(\nu / \lambda)$.

Proof. When $|\nu|<|\lambda|+|\mu|, c_{\lambda, \mu}^{\nu}=0$ for geometric reasons. Clearly in this case also $\mathrm{QBallot}_{\mu}(\nu / \lambda)=\emptyset$.

Suppose $|\nu|=|\lambda|+|\mu|$ and $T \in \operatorname{QBallot}_{\mu}(\nu / \lambda)$. The number of boxes of $\nu / \lambda$ on the main diagonal is $\ell(\nu)-\ell(\lambda)$. By pigeonhole, each gene of $T$ is a single box. Hence these tableaux are exactly the tableaux of [St89, Theorem 8.3] with condition (2) removed. Therefore by the discussion of [St89, p. 126], \#QBallot ${ }_{\mu}(\nu / \lambda)$ is the coefficient of the Schur $Q$-function $Q_{\mu}$ in the expansion of the skew Schur $Q$-function $Q_{\nu / \lambda}$. It is well known that these coefficients agree with the structure constants for $Z$ in this case.

That is, we conjecturally have combinatorially-related upper and lower bounds for $\left|c_{\lambda, \mu}^{\nu}\right|$ in terms of genomic tableaux. Let

$\operatorname{PBallot}_{\mu}(\nu / \lambda):=\{$ ballot genomic $P$-tableaux of shape $\nu / \lambda$ with content $\mu\}$.

Naturally, one seeks a set QBallot ${ }_{\mu}^{\star}(\nu / \lambda)$ satisfying

$$
\operatorname{PBallot}_{\mu}(\nu / \lambda) \subseteq \mathrm{QBallot}_{\mu}^{\star}(\nu / \lambda) \subseteq \mathrm{QBallot}_{\mu}(\nu / \lambda),
$$

such that \#QBallot ${ }_{\mu}^{\star}(\nu / \lambda)=\left|c_{\lambda, \mu}^{\nu}\right|$. Let

QBallot ${ }_{\mu}^{\dagger}(\nu / \lambda):=$

$\left\{T \in \mathrm{QBallot}_{\mu}(\nu / \lambda)\right.$ : no gene contains both primed and unprimed labels $\}$.

Conjecture 8.8. \#QBallot ${ }_{\mu}^{\dagger}(\nu / \lambda) \leq\left|c_{\lambda, \mu}^{\nu}\right|$.

This has also been computer-checked for $n \leq 6$. It suggests that one should look to define QBallot ${ }_{\mu}^{\star}(\nu / \lambda)$ from QBallot ${ }_{\mu}(\nu / \lambda)$ by imposing a condition on genes with both primed and unprimed labels. 


\section{Proof of OG Genomic Littlewood-Richardson Rule (Theorem 8.1)}

Our proof of Theorem 8.1 proceeds parallel to the first proof of Theorem 1.4. (We are not aware of any set-valued tableau or puzzle formulation of Theorem 8.1.)

9.1. Shifted $K$-(semi)standardization maps. Let $T$ be a genomic $P$-tableau. Impose a total order on genes of $T$ by $\mathcal{G}_{1}<\mathcal{G}_{2}$ if $\mathrm{b}_{1} \prec \mathrm{b}_{2}$, for $\mathrm{b}_{i}$ a box of $\mathcal{G}_{i}$. (Note that since the boxes of a gene form a $\prec$-interval, this order is well-defined.)

A shifted increasing tableau is a filling of a shifted shape that strictly increases along rows and down columns (see [ThYo09b, §7] and [ClThYo14]). Define the shifted $K$ standardization map

$$
\Gamma: \operatorname{PGen}_{\mu}(\nu / \lambda) \rightarrow \operatorname{Inc}(\nu / \lambda)
$$

by filling the $i$ th gene in $<-$ order with the entry $i$.

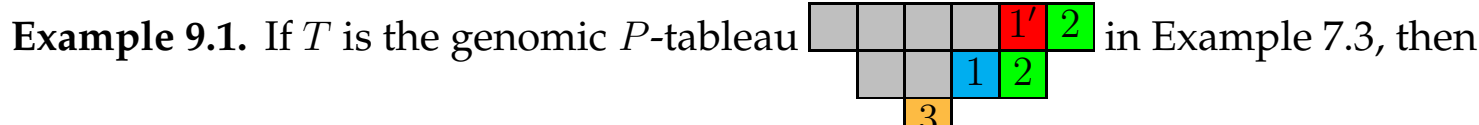

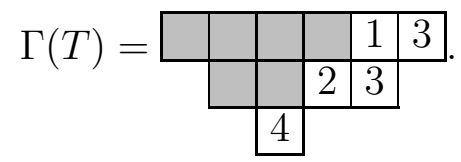

Recall

$$
\mathcal{P}_{k}(\mu):=\left\{1+\sum_{i<k} \mu_{i}, 2+\sum_{i<k} \mu_{i}, \ldots, \sum_{j \leq k} \mu_{j}\right\} .
$$

and let $S \in \operatorname{Inc}(\nu / \lambda)$ have largest entry $n$. Let

$$
\mu=\left(\mu_{1}, \mu_{2}, \ldots, \mu_{h}\right)
$$

be a composition of $n$. The shifted $K$-semistandardization $\Delta_{\mu}(S)$ with respect to $\mu$ is defined as follows. Replace each entry $i$ in $S$ with $k_{i}$ for the unique $k$ such that $i \in \mathcal{P}_{k}(\mu)$. For each $k_{h}$, replace it with $k^{\prime}$ if there is a $k_{j}$ southwest of it with $h<j$; otherwise replace it with $k$. If the result is a $P$-tableau, define a (putative) genomic $P$-tableau structure by putting all boxes that have the same entry in $S$ into the same gene. If the result is a $P$ genomic tableau, we say $\mu$ is admissible for $S$; otherwise $\Delta_{\mu}(S)$ is not defined. Clearly, if $\Delta_{\mu}(S)$ is defined, it has content $\mu$.

Example 9.2. Let $S$ be the increasing tableau of Example 9.1. Let $\eta=(2,1,1)$. We compute $\Delta_{\eta}(S)$ in stages:

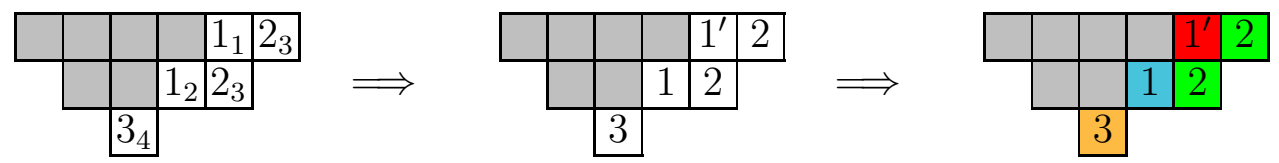

Observe that we obtain the genomic $P$-tableau $T$ of Example 7.3 . 
Compare this to the computation of $\Delta_{\theta}(S)$, where $\theta=(4)$ :

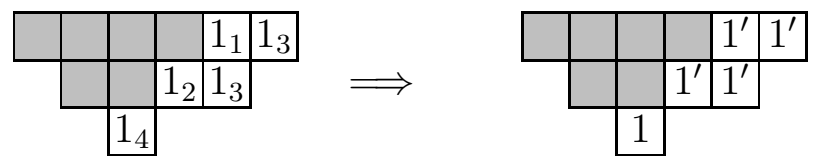

Since the tableau obtained is not a $P$-tableau (it violates (P.3)), $\Delta_{\theta}(S)$ is undefined.

Example 9.3. Let $V$ be the increasing tableau \begin{tabular}{l|l|l|}
\hline & & 1 \\
\hline
\end{tabular} and let $\kappa=(2)$. Then in the construction of $\Delta_{\kappa}(V)$, we first obtain a valid $P$-tableau:

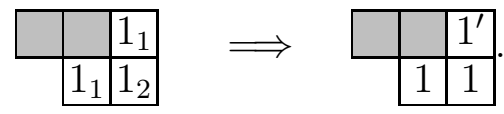

However the putative genomic structure

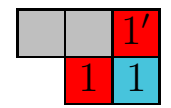

is invalid, so $\Delta_{\kappa}(V)$ is undefined.

An increasing tableau $S$ is $\mu$-Pieri-filled if $\mu$ is admissible for $S$ and $\Gamma\left(\Delta_{\mu}(S)\right)=S$.

Remark 9.4. It is easy to check that for $\mu=\left(\mu_{1}, \mu_{2}, \ldots, \mu_{h}\right)$, an increasing tableau $S$ is $\mu$-Pieri-filled if and only if for each $k \leq h$, the entries of $S$ in $\mathcal{P}_{k}(\mu)$ form a Pieri filling of a ribbon in the sense of [ClThYo14, §4].

Lemma 9.5. Let $T \in \operatorname{PGen}_{\mu}(\nu / \lambda)$. Then $\mu$ is admissible for $\Gamma(T)$ and $\Delta_{\mu}(\Gamma(T))=T$. Hence $\Gamma(T)$ is $\mu$-Pieri-filled.

Proof. The construction of $\Delta_{\mu}(\Gamma(T))$ is in stages. First we construct the underlying putative $P$-tableau structure for $\Delta_{\mu}(\Gamma(T))$. We will show that this is the same as the underlying $P$-tableau of $T$. Consider a box $\mathrm{b}$ in $\nu / \lambda$. Suppose the box b in $T$ contains $\alpha \in \mathcal{D}$ (the color being irrelevant for now). Then it is clear that $\Delta_{\mu}(\Gamma(T))$ has $\beta \in \mathrm{b}$ with $|\beta|=|\alpha|$. The letter $\beta$ is primed if and only if there is $\gamma$ in box c southwest of b in $\Delta_{\mu}(\Gamma(T))$ with $|\gamma|=|\beta|$ and the entry of $\mathrm{c}$ in $\Gamma(T)$ strictly greater than the entry of b in $\Gamma(T)$. The entry of $\mathrm{c}$ in $\Gamma(T)$ is strictly greater than the entry of $\mathrm{b}$ in $\Gamma(T)$ exactly when $\mathrm{b} \prec \mathrm{c}$. By definition, this happens if and only if $\alpha$ is primed. Thus $\alpha=\beta$. Therefore $T$ and (the partially constructed tableau) $\Delta_{\mu}(\Gamma(T))$ have the same underlying $P$-tableau structure.

In the next stage of constructing $\Delta_{\mu}(\Gamma(T))$, we attempt to partition the boxes into genes to produce a genomic $P$-tableau. By construction, $T$ and $\Delta_{\mu}(\Gamma(T))$ have the same partition of labels into genes; hence $\Delta_{\mu}(\Gamma(T))$ is defined and the first claim of the lemma holds. The second claim follows from the first by applying $\Gamma$.

Let $\mathrm{PF}_{\mu}(\nu / \lambda):=\{S: S$ is increasing of shape $\nu / \lambda$ and $\mu$-Pieri filled $\}$.

Theorem 9.6. $\Gamma: \operatorname{PGen}_{\mu}(\nu / \lambda) \rightarrow \mathrm{PF}_{\mu}(\nu / \lambda)$ and $\Delta_{\mu}: \mathrm{PF}_{\mu}(\nu / \lambda) \rightarrow \mathrm{PGen}_{\mu}(\nu / \lambda)$ are mutually inverse bijections.

Proof. Immediate by definition and Lemma 9.5 . 
9.2. Genomic $P$-Knuth equivalence. Given a colored sequence $w$ of symbols from $\mathcal{D}$, write $\hat{w}$ for the sequence given by writing $w$ backwards, replacing each $k^{\prime}$ with $k$ and each $k$ with $(k+1)^{\prime}$ and preserving the colors (cf. the uncolored definition of $\hat{w}$ after Example (7.3). A genomic $P$-word is a word $s$ of colored symbols from $\mathcal{D}$ such that in the concatenation $s \hat{s}$ all unprimed $i$ 's of a fixed color are consecutive among the set of all unprimed $i$ 's. Let genomicseq $(T)$ denote the colored row reading word (right to left, and top to bottom) of a genomic $P$-tableau $T$, as for genomic tableaux in Section 3 ,

Lemma 9.7. Let $T$ be a genomic P-tableau. Then genomicseq $(T)$ is a genomic P-word.

Proof. The follows from the fact that $T$ is a $P$-tableau together with the condition that the boxes of each gene of family $i$ are consecutive in $\prec$-order.

A genotype of a genomic $P$-word $w$ is an uncolored subword given by choosing one letter of each color. A $P$-genotype of the double sequence $w \hat{w}$ is a word of the form $x \hat{x}$ where $x$ is any genotype of $w$. We say $w \hat{w}$ is locally ballot at the letter $\alpha$ if every $P$ genotype of $w \hat{w}$ that includes that $\alpha$ is locally ballot there. Finally $w \hat{w}$ is ballot if every $P$-genotype of $w \hat{w}$ is ballot, equivalently if $w \hat{w}$ is locally ballot at each letter. In particular, the genomic $P$-tableau $T$ is ballot exactly when genomicseq $(T)$ genomicseq $(T)$ is.

Example 9.8. Let $T$ be the genomic $P$-tableau \begin{tabular}{l|l|l|l|l}
\hline & & & $1^{\prime}$ & 2 \\
& & & 1 & 2 \\
& 3 &
\end{tabular} of Example7.3, Then

$$
\text { genomicseq }(T) \widehat{\text { genomicseq }}(T)=21^{\prime} 2134^{\prime} 2^{\prime} 3^{\prime} 13^{\prime} \text {. }
$$

It has exactly two $P$-genotypes:

$$
21^{\prime} 134^{\prime} 2^{\prime} 13^{\prime} \text { and } 1^{\prime} 2134^{\prime} 2^{\prime} 3^{\prime} 1 .
$$

Neither P-genotype is ballot.

We define the equivalence relation $\equiv_{G P}$ of genomic $P$-Knuth equivalence on genomic $P$-words as the transitive closure of the following relations:

$$
\begin{aligned}
\mathbf{u} \alpha \alpha \mathbf{v} & \equiv_{G P} \mathbf{u} \alpha \mathbf{v}, \\
\mathbf{u} \alpha \beta \alpha \mathbf{v} & \equiv_{G P} \mathbf{u} \alpha \beta \mathbf{v}, \\
\mathbf{u} \beta \alpha \gamma \mathbf{v} & \equiv_{G P} \mathbf{u} \beta \gamma \mathbf{v} \text { if } \alpha \leq \beta<\gamma \text { and } \beta=|\beta|, \text { or } \alpha<\beta \leq \gamma \text { and } \beta=|\beta|^{\prime}, \\
\mathbf{u} \alpha \gamma \beta \mathbf{v} & \equiv_{G P} \mathbf{u} \gamma \beta \mathbf{v} \text { if } \alpha \leq \beta<\gamma \text { and } \beta=|\beta|^{\prime}, \text { or } \alpha<\beta \leq \gamma \text { and } \beta=|\beta|, \\
\mathbf{u} i j & \equiv_{G P} \mathbf{u} j^{\dagger} i, \text { where } j^{\dagger}=j^{\prime} \text { if } i=j, \text { and } j^{\dagger}=j \text { otherwise, }
\end{aligned}
$$

where red, blue, green represent distinct colors.

Theorem 9.9. If $w_{1} \equiv_{G P} w_{2}$, then $w_{1} \hat{w}_{1}$ is ballot if and only if $w_{2} \hat{w}_{2}$ is ballot.

Proof. Let $w$ be a genomic $P$-word. We need that (GP.1)-(GP.5) preserve ballotness of $w \hat{w}$. (GP.1) and (GP.2): These relations change $w$ without changing the set of genotypes of $w \hat{w}$. Hence they do not affect ballotness of the latter.

(GP.3): Suppose $w=\mathbf{u} \beta \alpha \gamma \mathbf{v}$ and that $w^{*}=\mathbf{u} \beta \gamma \alpha \mathbf{v}$ is obtained by (GP.3).

(" $\Rightarrow$ " for (GP.3)): We assume $w \hat{w}$ is ballot, and we must show $w^{*} \hat{w}^{*}$ is ballot, i.e., locally ballot (henceforth abbreviated "LB") at each letter. 
(Case 1: $\alpha=\beta$ ): We have $i:=|\alpha|=\alpha$ and $i<\gamma$.

(Case 1.1: $\gamma=|\gamma|)$ : Let $k:=\gamma$. Then

$$
w \hat{w}=\mathbf{u} i i k \mathbf{v} \hat{\mathbf{v}}(k+1)^{\prime}(i+1)^{\prime}(i+1)^{\prime} \hat{\mathbf{u}}
$$

and

$$
w^{*} \hat{w}^{*}=\mathbf{u} i k i \mathbf{v} \hat{\mathbf{v}}(i+1)^{\prime}(k+1)^{\prime}(i+1)^{\prime} \hat{\mathbf{u}} .
$$

It suffices to show that $w^{*} \hat{w}^{*}$ is LB at $k$ and $(k+1)^{\prime}$. LBness at the latter is clear from the ballotness of $w \hat{w}$.

If $k>i+1$, then LBness at $k$ is also clear from the ballotness of $w \hat{w}$. Hence assume $k=i+1$. The proof is now the same is for the corresponding case of Theorem 3.5.

(Case 1.2: $\gamma=|\gamma|^{\prime}$ ): Let $k^{\prime}:=\gamma$. Then

$$
w \hat{w}=\mathbf{u} i i k^{\prime} \mathbf{v} \hat{\mathbf{v}} k(i+1)^{\prime}(i+1)^{\prime} \hat{\mathbf{u}}
$$

and

$$
w^{*} \hat{w}^{*}=\mathbf{u} i k^{\prime} i \mathbf{v} \hat{\mathbf{v}}(i+1)^{\prime} k(i+1)^{\prime} \hat{\mathbf{u}} .
$$

It suffices to show that $w^{*} \hat{w}^{*}$ is LB at $k^{\prime}$ and $k$. LBness at the latter is clear from the ballotness of $w \hat{w}$. LBness at the former may be argued exactly as in Case 1.1.

(Case 2: $\beta=\gamma$ ): We have $j^{\prime}:=|\beta|^{\prime}=\beta$ and $\alpha<j^{\prime}$.

(Case 2.1: $\alpha=|\alpha|)$ : Let $i=\alpha$. Then

$$
w \hat{w}=\mathbf{u} j^{\prime} i j^{\prime} \mathbf{v} \hat{\mathbf{v}} j(i+1)^{\prime} j \hat{\mathbf{u}}
$$

and

$$
w^{*} \hat{w}^{*}=\mathbf{u} j^{\prime} j^{\prime} i \mathbf{v} \hat{\mathbf{v}}(i+1)^{\prime} j j \hat{\mathbf{u}} .
$$

It suffices to show that $w^{*} \hat{w}^{*}$ is LB at $j^{\prime}$ and $j$. That $w^{*} \hat{w}^{*}$ is LB at $j^{\prime}$ follows from the LBness of $w \hat{w}$ at $j^{\prime}$. LBness at $j$ is trivial.

(Case 2.2: $\left.\alpha=|\alpha|^{\prime}\right)$ : Let $i^{\prime}=\alpha$. Then

$$
w \hat{w}=\mathbf{u} j^{\prime} i^{\prime} j^{\prime} \mathbf{v} \hat{\mathbf{v}} j i j \hat{\mathbf{u}}
$$

and

$$
w^{*} \hat{w}^{*}=\mathbf{u} j^{\prime} j^{\prime} i^{\prime} \mathbf{v} \hat{\mathbf{v}} i j j \hat{\mathbf{u}} .
$$

It suffices to check that $w^{*} \hat{w}^{*}$ is LB at $j^{\prime}$ and $j$. This is clear from the ballotness of $w \hat{w}$.

(Case 3: $\alpha<\beta<\gamma$ ):

(Case 3.1: $\alpha=|\alpha|$ ): Let $i=\alpha$. If $\gamma>i+1$, ballotness is clear. Otherwise, by the assumptions of Case 3, $\beta=(i+1)^{\prime}$ and $\gamma=i+1$. So

$$
w \hat{w}=\mathbf{u}(i+1)^{\prime} i(i+1) \mathbf{v} \hat{\mathbf{v}}(i+2)^{\prime}(i+1)^{\prime}(i+1) \hat{\mathbf{u}}
$$

and

$$
w^{*} \hat{w}^{*}=\mathbf{u}(i+1)^{\prime}(i+1) i \mathbf{v} \hat{\mathbf{v}}(i+1)^{\prime}(i+2)^{\prime}(i+1) \hat{\mathbf{u}} .
$$

It suffices to check that $w^{*} \hat{w}^{*}$ is LB at $(i+1)$ and $(i+2)^{\prime}$. The latter is clear from ballotness of $w \hat{w}$. The LBness at $(i+1)$ follows from the LBness of $w \hat{w}$ at $(i+1)^{\prime}$.

(Case 3.2: $\alpha=|\alpha|^{\prime}$ ): Let $i^{\prime}=\alpha$. If $\gamma>i+1$, ballotness is clear. Otherwise we have either $\gamma=(i+1)^{\prime}$ or $\gamma=i+1$.

(Case 3.2.1: $\left.\gamma=(i+1)^{\prime}\right)$ : We have $\beta=i$. Then

$$
w \hat{w}=\mathbf{u} i i^{\prime}(i+1)^{\prime} \mathbf{v} \hat{\mathbf{v}}(i+1) i(i+1)^{\prime} \hat{\mathbf{u}}
$$


and

$$
w^{*} \hat{w}^{*}=\mathbf{u} i(i+1)^{\prime} i^{\prime} \mathbf{v} \hat{\mathbf{v}} i(i+1)(i+1)^{\prime} \hat{\mathbf{u}} .
$$

It suffices to check LBness at the two green letters. These checks hold by the ballotness of $w \hat{w}$.

(Case 3.2.2: $\gamma=i+1)$ : Here

$$
w \hat{w}=\mathbf{u} \beta i^{\prime}(i+1) \mathbf{v} \hat{\mathbf{v}}(i+2)^{\prime} i \hat{\beta} \hat{\mathbf{u}}
$$

and

$$
w^{*} \hat{w}^{*}=\mathbf{u} \beta(i+1) i^{\prime} \mathbf{v} \hat{\mathbf{v}} i(i+2)^{\prime} \hat{\beta} \hat{\mathbf{u}} .
$$

It suffices to check LBness at the two green letters. These checks are both direct from the ballotness of $w \hat{w}$.

(" $\Leftarrow "$ for (GP.3)): Conversely, assume $w^{*} \hat{w}^{*}$ is ballot. We need to show that $w \hat{w}$ is ballot. As with the arguments for $\Rightarrow$, we need to establish LBness at each letter. In brief, it suffices to check this in each case below at the green letters. In each of these situations, this is immediate from the assumption $w^{*} \hat{w}^{*}$ is ballot.

(Case 1: $\alpha=\beta$ ): We have $i:=|\alpha|=\alpha$ and $i<\gamma$.

(Case 1.1: $\gamma=|\gamma|$ ): Let $k:=\gamma$. Then

$$
w^{*} \hat{w}^{*}=\mathbf{u} i k i \mathbf{v} \hat{\mathbf{v}}(i+1)^{\prime}(k+1)^{\prime}(i+1)^{\prime} \hat{\mathbf{u}}
$$

and

$$
w \hat{w}=\mathbf{u} i i k \mathbf{v} \hat{\mathbf{v}}(k+1)^{\prime}(i+1)^{\prime}(i+1)^{\prime} \hat{\mathbf{u}} .
$$

(Case 1.2: $\gamma=|\gamma|^{\prime}$ ): Let $k^{\prime}:=\gamma$. Then

$$
w^{*} \hat{w}^{*}=\mathbf{u} i k^{\prime} i \mathbf{v} \hat{\mathbf{v}}(i+1)^{\prime} k(i+1)^{\prime} \hat{\mathbf{u}}
$$

and

$$
w \hat{w}=\mathbf{u} i i k^{\prime} \mathbf{v} \hat{\mathbf{v}} k(i+1)^{\prime}(i+1)^{\prime} \hat{\mathbf{u}} .
$$

(Case 2: $\beta=\gamma$ ): We have $j^{\prime}:=|\beta|^{\prime}=\beta$ and $\alpha<j^{\prime}$.

(Case 2.1: $\alpha=|\alpha|$ ): Let $i=\alpha$. Then

$$
w^{*} \hat{w}^{*}=\mathbf{u} j^{\prime} j^{\prime} i \mathbf{v} \hat{\mathbf{v}}(i+1)^{\prime} j j \hat{\mathbf{u}}
$$

and

$$
w \hat{w}=\mathbf{u} j^{\prime} i j^{\prime} \mathbf{v} \hat{\mathbf{v}} j(i+1)^{\prime} j \hat{\mathbf{u}} .
$$

(Case 2.2: $\alpha=|\alpha|^{\prime}$ ): Let $i^{\prime}=\alpha$. Then

$$
w^{*} \hat{w}^{*}=\mathbf{u} j^{\prime} j^{\prime} i^{\prime} \mathbf{v} \hat{\mathbf{v}} i j j \hat{\mathbf{u}}
$$

and

$$
w \hat{w}=\mathbf{u} j^{\prime} i^{\prime} j^{\prime} \mathbf{v} \hat{\mathbf{v}} j i j \hat{\mathbf{u}} .
$$

(Case 3: $\alpha<\beta<\gamma$ ):

(Case 3.1: $\alpha=|\alpha|$ ): Let $i=\alpha$. If $\gamma>i+1$, ballotness is clear. Otherwise, by the assumptions of Case 3, we have $\beta=(i+1)^{\prime}$ and $\gamma=i+1$. Thus

$$
w^{*} \hat{w}^{*}=\mathbf{u}(i+1)^{\prime}(i+1) i \mathbf{v} \hat{\mathbf{v}}(i+1)^{\prime}(i+2)^{\prime}(i+1) \hat{\mathbf{u}}
$$

and

$$
w \hat{w}=\mathbf{u}(i+1)^{\prime} i(i+1) \mathbf{v} \hat{\mathbf{v}}(i+2)^{\prime}(i+1)^{\prime}(i+1) \hat{\mathbf{u}} .
$$


(Case 3.2: $\alpha=|\alpha|^{\prime}$ ): Let $i^{\prime}=\alpha$. If $\gamma>i+1$, ballotness is clear. Otherwise we have either $\gamma=(i+1)^{\prime}$ or $\gamma=i+1$.

(Case 3.2.1: $\left.\gamma=(i+1)^{\prime}\right)$ : We have $\beta=i$. Then

$$
w^{*} \hat{w}^{*}=\mathbf{u} i(i+1)^{\prime} i^{\prime} \mathbf{v} \hat{\mathbf{v}} i(i+1)(i+1)^{\prime} \hat{\mathbf{u}}
$$

and

$$
w \hat{w}=\mathbf{u} i i^{\prime}(i+1)^{\prime} \mathbf{v} \hat{\mathbf{v}}(i+1) i(i+1)^{\prime} \hat{\mathbf{u}} .
$$

(Case 3.2.2: $\gamma=i+1$ ): Here

$$
w^{*} \hat{w}^{*}=\mathbf{u} \beta(i+1) i^{\prime} \mathbf{v} \hat{\mathbf{v}} i(i+2)^{\prime} \hat{\beta} \hat{\mathbf{u}}
$$

and

$$
w \hat{w}=\mathbf{u} \beta i^{\prime}(i+1) \mathbf{v} \hat{\mathbf{v}}(i+2)^{\prime} i \hat{\beta} \hat{\mathbf{u}} .
$$

(GP.4): This may be argued exactly as for (GP.3).

(GP.5): Suppose $w=\mathbf{u} i j$ and that $w^{*}=\mathbf{u} j^{\dagger} i$ is obtained by (GP.5). By symmetry, we may assume $i \leq j$. We must show $w \hat{w}$ is ballot if and only if $w^{*} \widehat{w^{*}}$ is.

(Case 1: $i<j$ ): Then

$$
w \hat{w}=\mathbf{u} i j(j+1)^{\prime}(i+1)^{\prime} \hat{\mathbf{u}},
$$

while

$$
w^{*} \widehat{w^{*}}=\mathbf{u} j i(i+1)^{\prime}(j+1)^{\prime} \hat{\mathbf{u}} .
$$

Suppose $w \hat{w}$ is ballot. It suffices to check LBness of $w^{*} \widehat{w^{*}}$ at the two blue letters. LBness at $(j+1)^{\prime}$ is clear from the assumed ballotness of $w \hat{w}$. LBness at $j$, for $j=i+1$, follows from the LBness of $w \hat{w}$ at $(i+1)^{\prime}$ (when $j \neq i+1$, the claim is clear).

Conversely suppose $w^{*} \widehat{w^{*}}$ is ballot. It suffices to check LBness of $w \hat{w}$ at the two blue letters; this is immediate.

(Case 2: $i=j$ ): Then

$$
w \hat{w}=\mathbf{u} i i(i+1)^{\prime}(i+1)^{\prime} \hat{\mathbf{u}},
$$

while

$$
w^{*} \widehat{w^{*}}=\mathbf{u}^{\prime} i(i+1)^{\prime} i \hat{\mathbf{u}} .
$$

It is straightforward that $w \hat{w}$ is ballot if and only if $w^{*} \widehat{w^{*}}$ is.

Weak $K$-Knuth equivalence on words is the symmetric, transitive closure of these relations [BuSa13, Definition 7.6]:

$$
\begin{aligned}
\mathbf{u} a a \mathbf{v} & \equiv_{w K} \mathbf{u} a \mathbf{v}, \\
\mathbf{u} a b a \mathbf{v} & \equiv_{w K} \mathbf{u} b a b \mathbf{v}, \\
\mathbf{u} b a c \mathbf{v} & \equiv_{w K} \mathbf{u} b c a \mathbf{v} \text { if } a<b<c, \\
\mathbf{u} a c b \mathbf{v} & \equiv_{w K} \mathbf{u} c a b \mathbf{v} \text { if } a<b<c, \\
\mathbf{u} a b & \equiv_{w K} \mathbf{u} b a .
\end{aligned}
$$

Lemma 9.10. For genomic $P$-words $u, v$ we have $u \equiv_{G P} v$ if and only if $\Gamma(u) \equiv_{w K} \Gamma(v)$.

Proof. This follows from applying $\Delta_{\mu}$ to the generating relations for weak $K$-Knuth equivalence for Pieri-filled words. 
9.3. Shifted jeu de taquin and the conclusion of the proof. The definitions of genomic jeu de taquin and $K$-jeu de taquin for shifted tableaux are analogous to the unshifted case. For details of shifted $K$-jeu de taquin, see [ClThYo14]. We sketch the modifications necessary for shifted genomic jeu de taquin and give an illustrative example. For each gene $\mathcal{G}$ of family $k$, define the operator $\operatorname{switch}_{\mathcal{G}}^{\bullet}$ as follows: If b is a box of $\mathcal{G}$ in the tableau $T$ with a neighbor containing a $\bullet$, replace the $k$ or $k^{\prime} \in \mathrm{b}$ with $\bullet$ and remove it from $\mathcal{G}$. If $\mathrm{c}$ is a box of $T$ containing a $\bullet$ and with a $\mathcal{G}$ neighbor, c is a box of $\mathcal{G}$ in $\operatorname{switch}_{\mathcal{G}}^{\bullet}(T)$; c has entry $k$ in $\operatorname{switch}_{\mathcal{G}}^{\bullet}(T)$ if either of its $\mathcal{G}$ neighbors in $T$ have entry $k$ or if c lies on the main diagonal; otherwise c has entry $k^{\prime}$ in $\operatorname{switch}_{\mathcal{G}}^{\bullet}(T)$. The other boxes of $T$ are the same in $\operatorname{switch}_{\mathcal{G}}^{*}(T)$.

Index the genes of $T$ as

$$
\mathcal{G}_{1}<\mathcal{G}_{2}<\cdots<\mathcal{G}_{|\mu|}
$$

according to the total order on genes from Section 9.1. Then

$$
\operatorname{jdt}_{I}(T):=\operatorname{switch}_{\mathcal{G}_{|\mu|}}^{\bullet} \circ \cdots \circ \operatorname{switch}_{\mathcal{G}_{2}}^{\bullet} \circ \operatorname{switch}_{\dot{\mathcal{G}}_{1}}\left(T^{\bullet}\right)
$$

with the $\bullet$ 's deleted. (This algorithm reduces to the classical jeu de taquin for semistandard $P$-tableaux in the case each gene contains only a single box.)

Example 9.11. Suppose $T^{\bullet}$ is the genomic tableau $\left.\begin{array}{l|l|l|l}\hline & \bullet & 1^{\prime} & 1 \\ \bullet & 1^{\prime} & 2 \\ \hline\end{array}\right]$. Then

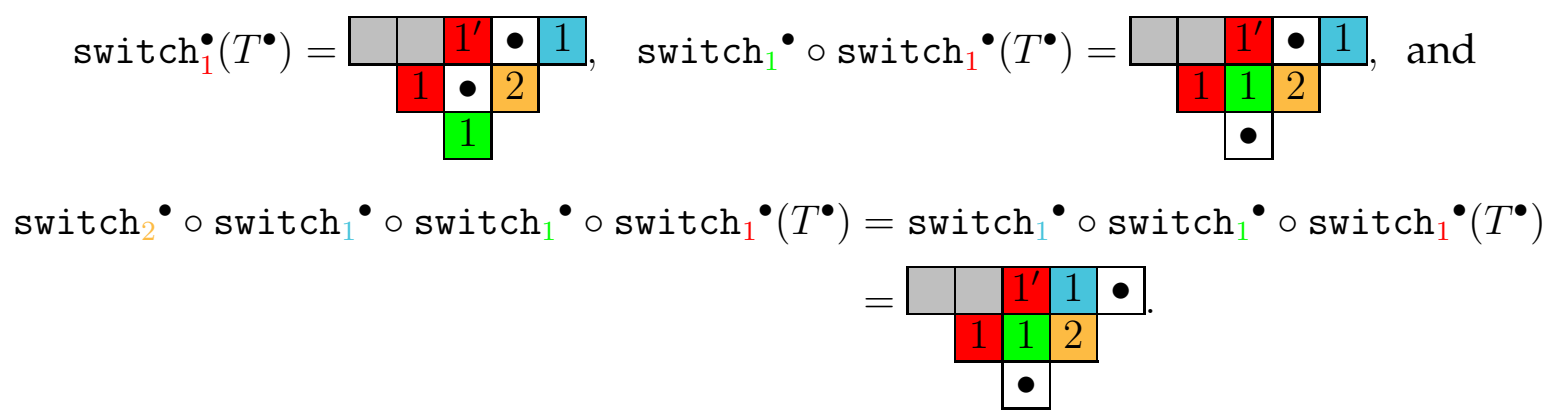

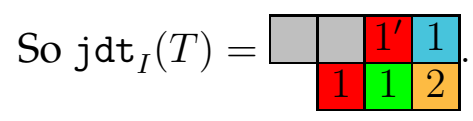

Using this shifted genomic jeu de taquin, one can obtain shifted versions of genomic infusion and genomic Bender-Knuth involutions, analogous to the discussion of Section 6 , This leads to a definition of genomic P-Schur functions, symmetric functions that deform the classical P-Schur functions just as the genomic Schur functions of Section 6 deform the classical Schur functions. We do not pursue these ideas further here.

Let $S_{\mu}$ denote the row superstandard tableau of shifted shape $\mu$ (that is, the tableau whose first row has entries $1,2,3, \ldots, \mu_{1}$, and whose second row has entries $\mu_{1}+1, \mu_{2}+$ $2, \ldots, \mu_{1}+\mu_{2}$ etc.).

Example 9.12. For $\mu=(4,2), S_{\mu}=$\begin{tabular}{|l|l|l|l|}
\hline & 2 & 3 & 4 \\
\hline & 5 & 6
\end{tabular}.

Let

$$
T_{\mu}:=\Delta_{\mu}\left(S_{\mu}\right)
$$


be the unique genomic $P$-tableau whose underlying $P$-tableau is the highest weight tableau of shifted shape $\mu$. We recall some results that we need.

Theorem 9.13 ([BuSa13, Theorem 7.8]). Let $S$ be a shifted increasing tableaux. Then $S$ rectifies to $S_{\mu}$ if and only if $\operatorname{seq}(S) \equiv_{w K} \operatorname{seq}\left(S_{\mu}\right)$.

Let $\operatorname{IncRect}_{\mu}(\nu / \lambda):=\left\{\right.$ shifted increasing tableaux of shape $\nu / \lambda$ that rectify to $\left.S_{\mu}\right\}$.

Theorem 9.14 ([ClThYo14, Theorem 1.2]). $b_{\lambda, \mu}^{\nu}=(-1)^{|\nu|-|\lambda|-|\mu|} \times \# \operatorname{IncRect}_{\mu}(\nu / \lambda)$.

By Theorem 9.14, it is enough to biject $\operatorname{IncRect}_{\mu}(\nu / \lambda)$ and $\operatorname{PBallot}_{\mu}(\nu / \lambda)$. We claim that the maps $\Gamma$ and $\Delta_{\mu}$ give the desired bijections. It follows from Remark 9.4 and [ClThYo14, Proof of Theorem 1.1] that $\Delta_{\mu}$ is well-defined on $\operatorname{IncRect}_{\mu}(\nu / \lambda)$.

Let $S \in \operatorname{IncRect}_{\mu}(\nu / \lambda)$. By Theorem 9.13 ,

$$
\operatorname{seq}(S) \equiv_{w K} \operatorname{seq}\left(S_{\mu}\right) .
$$

By Lemma 9.10 ,

$$
\text { genomicseq }\left(\Delta_{\mu}(S)\right) \equiv_{G P} \text { genomicseq }\left(\Delta_{\mu}\left(S_{\mu}\right)\right)=\text { genomicseq }\left(T_{\mu}\right) \text {. }
$$

Note genomicseq $\left(T_{\mu}\right)$ is ballot. Hence by Theorem 9.9 , genomicseq $\left(\Delta_{\mu}(S)\right)$ is ballot. Thus

$$
\Delta_{\mu}(S) \in \mathrm{PBallot}_{\mu}(\nu / \lambda) \text {. }
$$

Conversely, if $T \in \mathrm{PBallot}_{\mu}(\nu / \lambda)$, then its genomic rectification is also ballot by Theorem 4.2. Hence its genomic rectification is $T_{\mu}$. Therefore

$$
\Gamma(T) \in \operatorname{IncRect}_{\mu}(\nu / \lambda) .
$$

This completes the proof.

\section{ACKNOWLEDGMENTS}

We thank Hugh Thomas for many conversations which helped to make this project possible. OP was supported by an NSF Graduate Research Fellowship, and Illinois Distinguished Fellowship from the University of Illinois, and NSF MCTP grant DMS 0838434. AY was supported by NSF grants and a Helen Corley Petit fellowship at UIUC.

\section{REFERENCES}

[BlPeSa16] J. Bloom, O. Pechenik and D. Saracino, Proofs and generalizations of a homomesy conjecture of Propp and Roby, Discrete Math. 339 (2016), 194-206.

[Br02] M. Brion, Positivity in the Grothendieck group of complex flag varieties, J. Algebra 258 (2002), 137-159.

[Br05] M. Brion, Lectures on the geometry of flag varieties, Topics in cohomological studies of algebraic varieties, 33-85, Trends Math., Birkhäuser, Basel, 2005.

[Bu02] A. Buch, A Littlewood-Richardson rule for the K-theory of Grassmannians, Acta Math. 189 (2002), 37-78.

[Bu05] A. Buch, Combinatorial K-theory, Topics in cohomological studies of algebraic varieties, 87103, Trends Math., Birkhäuser, Basel, 2005.

[BKSTY08] A. Buch, A. Kresch, M. Shimozono, H. Tamvakis and A. Yong, Stable Grothendieck polynomials and $K$-theoretic factor sequences, Math. Ann. 340 (2008), 359-382.

[BuRa12] A. Buch and V. Ravikumar, Pieri rules for the K-theory of cominuscule Grassmannians, J. Reine Angew. Math. (Crelle's J.) 668 (2012), 109-132. 
[BuSa13] A. Buch and M. Samuel, $K$-theory of minuscule varieties, J. Reine Angew. Math. (Crelle's J.), to appear, 2014. arXiv:1306.5419

[ClThYo14] E. Clifford, H. Thomas and A. Yong, K-theoretic Schubert calculus for $O G(n, 2 n+1)$ and jeu de taquin for shifted increasing tableaux, J. Reine Angew. Math. (Crelle's J.) 690 (2014), 51-63.

[CoVa05] I. Coşkun and R. Vakil, Geometric positivity in the cohomology of homogeneous spaces and generalized Schubert calculus, in "Algebraic Geometry — Seattle 2005" Part 1, 77-124, Proc. Sympos. Pure Math., 80, Amer. Math. Soc., Providence, RI, 2009.

[DiPeSt15] K. Dilks, O. Pechenik and J. Striker, Resonance in orbits of plane partitions and increasing tableaux, preprint, 2015. arXiv: 1512.00365

[FoKi94] S. Fomin and A. N. Kirillov, Grothendieck polynomials and the Yang-Baxter equation, Proc. 6th Intern. Conf. on Formal Power Series and Algebraic Combinatorics, DIMACS, 1994, 183190.

[Fu97] W. Fulton, Young tableaux: With applications to representation theory and geometry, London Mathematical Society Student Texts, 35, Cambridge University Press, Cambridge, 1997.

[GMPPRST16] C. Gaetz, M. Mastrianni, R. Patrias, H. Peck, C. Robichaux, D. Schwein and K. Tam, $K-$ Knuth equivalence for increasing tableaux, Electron. J. Combin. 23 (2016), 1-37.

[HKPWZZ15] Z. Hamaker, A. Keilthy, R. Patrias, L. Webster, Y. Zhang and S. Zhou, Shifted Hecke insertion and the $K$-theory of $\mathrm{OG}(n, 2 n+1)$, preprint, 2015. arXiv: 1510.08972

[HPPW16] Z. Hamaker, R. Patrias, O. Pechenik and N. Williams, Doppelgangers: Bijections of plane partitions, preprint, 2016. arXiv:1602.05535

[HoHu92] P. Hoffman and J. Humphreys, Projective representations of the symmetric group: Q-functions and shifted tableaux, Oxford Mathematical Monographs, Oxford University Press, New York, 1992.

[IkNa13] T. Ikeda and H. Naruse, $K$-theoretic analogue of factorial Schur P-and $Q$-functions, Adv. Math. 243 (2013), 22-66.

[IkNaNu11] T. Ikeda, H. Naruse and Y. Numata, Bumping algorithm for set-valued shifted tableaux, Discrete Math. Theor. Comput. Sci. Proc. AO (2011), 527-538. FPSAC 2011, Reykjavik, Iceland.

[Kn70] D. Knuth, Permutations, matrices, and generalized Young tableaux, Pacific J. Math. 34 (1970), 709-727.

[Kn14] A. Knutson, Schubert calculus and shifting of interval positroid varieties, preprint, 2014. arXiv: 1408.1261

[KnTa03] A. Knutson and T. Tao, Puzzles and (equivariant) cohomology of Grassmannians, Duke Math. J. 119 (2003), no. 2, 221-260.

[LaPy07] T. Lam and P. Pylyavskyy, Combinatorial Hopf algebras and K-homology of Grassmannians, Int. Math. Res. Not. (2007), no. 24.

[LaSc82] A. Lascoux and M. -P. Schützenberger, Structure de Hopf de l'anneau de cohomologie et de l'anneau de Grothendieck d'une variété de drapeaux, C. R. Acad. Sci. Paris, 295 (1982), 629-633.

[Le00] C. Lenart, Combinatorial aspects of the K-theory of Grassmannians, Ann. Comb. 4 (2000), 67-82.

[LiMoSh16] H. Li, J. Morse and P. Shields, Structure constants for K-theory of Grassmannians revisited, preprint, 2016. arXiv:1601.04509

[LiRi34] D. E. Littlewood and A. R. Richardson, Group characters and algebra, Philos. Trans. R. Soc. Lond. Ser. A Math. Phys. Eng. Sci. 233 (1934), 99-141.

[Mo16+] C. Monical, Combinatorial K-theoretic Demazure atoms, in preparation, 2016.

[Pa15]

[PaPy14]

R. Patrias, Antipode formulas for combinatorial Hopf algebras, preprint, 2015. arXiv:1501.00710

[PaPy16]

[Pe14]

R. Patrias and P. Pylyavskyy, Dual filtered graphs, preprint, 2014. arXiv:1410.7683.

R. Patrias and P. Pylyavskyy, Combinatorics of $K$-theory via a $K$-theoretic Poirier-Reutenauer bialgebra, Discrete Math., 339 (2016), 1095-1115.

[PeYo15a] O. Pechenik and A. Yong, Genomic tableaux and combinatorial K-theory, Discrete Math. Theor. Comput. Sci. Proc. FPSAC'15 (2015), 37-48. FPSAC 2015, Daejeon, South Korea.

[PeYo15b] O. Pechenik and A. Yong, Equivariant K-theory of Grassmannians, preprint, 2015. arXiv: 1506.01992

[PeYo15c] O. Pechenik and A. Yong, Equivariant K-theory of Grassmannians II: The Knutson-Vakil conjecture, preprint, 2015. arXiv:1508.00446 
[Pr89] P. Pragacz, Algebro-geometric applications of Schur S-and Q-polynomials, Topics in invariant theory (Paris, 1989/1990), 130-191, Lecture Notes in Math., Springer, Berlin, 1991.

[PrStVi14] T. Pressey, A. Stokke and T. Visentin, Increasing tableaux and generalized Narayana numbers, Ann. Comb., accepted, 2015. arXiv:1409.2841

[ReTeYo16] V. Reiner, B. Tenner and A. Yong, Poset edge densities, nearly reduced words and barely set-valued tableaux, preprint, 2016.

[Rh15] B. Rhoades, A skein action of the symmetric group on noncrossing partitions, preprint, 2015. arXiv: 1501.04680

[St89] J. Stembridge, Shifted tableaux and the projective representations of symmetric groups, Adv. Math. 74 (1989), 87-134.

[ThYo09a] H. Thomas and A. Yong, A combinatorial rule for (co)minuscule Schubert calculus, Adv. Math. 222 (2009), no. 2, 596-620.

[ThYo09b] H. Thomas and A. Yong, A jeu de taquin theory for increasing tableaux, with applications to $K$-theoretic Schubert calculus, Algebra Number Theory 3 (2009), 121-148.

[ThYo11] H. Thomas and A. Yong, Longest increasing subsequences, Plancherel-type measure and the Hecke insertion algorithm, Adv. in Appl. Math. 46 (2011), 610-642.

[ThYo13] H. Thomas and A. Yong, Equivariant Schubert calculus and jeu de taquin, Ann. Inst. Fourier (Grenoble), to appear, 2013. arXiv:1207.3209

[Va06] R. Vakil, A geometric Littlewood-Richardson rule, Ann. of Math., 164 (2006), 371-422.

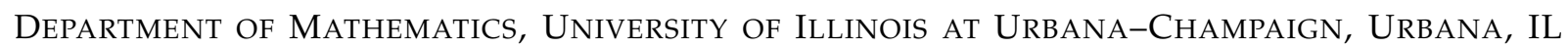
61801, USA

E-mail address: pecheni2@illinois.edu, ayong@uiuc.edu 